\title{
ANÁLISE DE SEQUENCIAS EQUILIBRADAS EM SÉRIE, DE TIPO I
}

\author{
GENEBALDO CORREIA FIGUEIREDO
}

Orientador: Dr. Izaias Rangel Nogueira

\begin{abstract}
Disserłação apresentada à Escola Superior de Agriculłura "Luiz de Queiroz", da Universidade de São Paulo, para obłenção do título de Mestre em Experimentação e Estatística.
\end{abstract}

PIRACI C A B A

Estado de São Paulo - Brasil

Outubro, 1978 
ii。

\section{A Olga, minha esposa, $e$ aos meus filhos Licia $e$ Genebaldo,}




\section{AGRADECIMENTOS}

Ao Dr. Izaias Rangel Nogueira, Chefe do Departamento de Matemática e Estatística da ESALQ, pela orientação geral do trabalho 。

Ao Dr. Bikas Kumar Sinha, Professor do Departamento de Estatística, do Instituto de Matemática da Universidade Federal da Bahia, pela sugestão do tema e pela excelente assisténcia cientí fica nos misteres que conduziram à realização desta pesquisa.

Ao Governo do Estado da Bahia, pela oportunidade ofe recida para a realização do curso.

Ao Dr. Frederico Pimentel Gomes, Coordenador do Curso de Pós-Graduação em Experimentação e Estatística, pela maneira sábia e amiga com que dirige o curso, como também pelas valiosas sugestões apresentadas.

Ao Dr. Raymundo Costa e Souza, Chefe do Departamento de Estatística, do Instituto de Matemática da Universidade Federal da Bahia, pelas importantes sugestões dadas.

Ao Dr. Cássio Roberto de Melo Godoi, Professor do De partamento de Matemática e Estatística da ESALQ, pela elaboração do Summary。

Ao Dr. Irundi Sampaio Edelweiss, Diretor Superintendente do Centro de Pesquisas e Desenvolvimento (CEPED), do Estado da Bahia, pelo apoio dado para a realização do curso. 
Ao CEPED e ao CNPq, pelo auxílio financeiro prestado.

Ao Corpo Docente do Departamento de Matemática e Estatística da ESALQ, pelos conhecimentos transmitidos, os quais concorreram, eficazmente, para a elaboração deste trabalho.

A Maria Izalina Ferreira Alves, Secretária do Departamento de Matemática e Estatística da ESALQ, pela amizade e espíri to de solidariedade.

Aos funcionários do Departamento de Matemática e Estatística da ESALQ, pela dedicação e atenção dispensada.

Aos colegas do Curso de Pós-Graduação em Experimenta ção e Estatística, pelo companheirismo.

A todos que concorreram para a realização do curso e deste trabalho. 


\section{INDICE}

Pág。

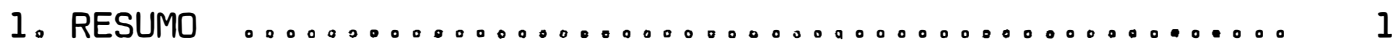

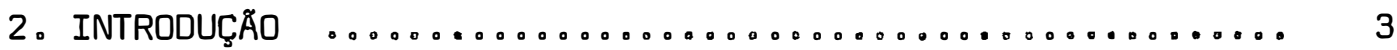

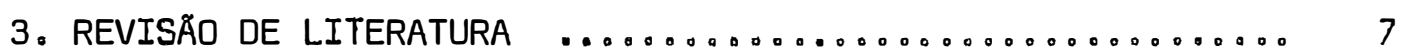

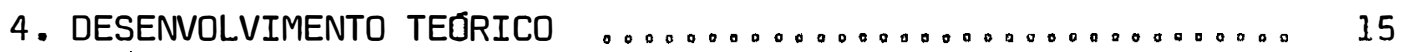

4.1 - Análise de Variância .................... 16

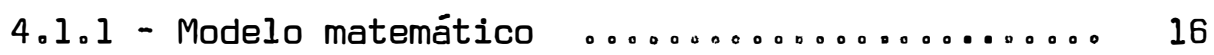

4.1 .2 - Sistema de equações normais $\ldots \ldots \ldots \ldots \ldots 17$

4.1 .3 - Estimadores dos parâmetros .............. 19

4.1 .4 - Esquema de análise de variância .......... 22

4.2 - Variâncias das Estimativas da Diferença Entre Dois

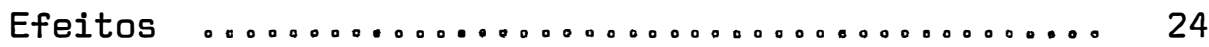

4.2.1 - Variância da estimativa da diferença entre dois efeitos diretos de tratamentos ...... 24

4.2.2 - Variância da estimativa da diferença entre dois efeitos residuais de tratamentos ..... 24

4.2.3 - Variância média da estimativa da diferença entre dois efeitos residuais de tratamentos 33

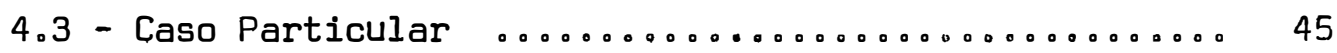

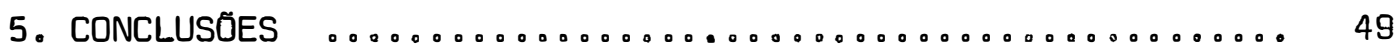

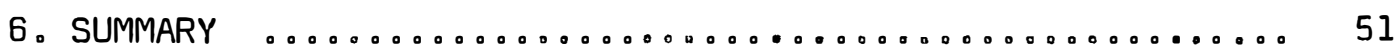

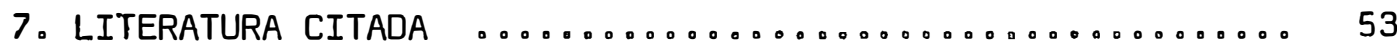




\section{RESUMO}

As Sequências Equilibradas em Série permitem estudar os efeitos diretos e residuais dos tratamentos aplicados, em períodos sucessivos, a uma unidade experimental.

A fim de se obterem resultados, através dos quais se possa analisar a classe das sequências especificadas neste trabalho, considera-se o modelo matemätico

$$
y_{h i j}=\mu+\beta_{h i}+\tau_{j}+\rho_{\ell(h, i, j)}+\varepsilon_{h i j} \text {, }
$$

onde: $y_{\text {hij }}$ é a observação referente ao tratamento $\underline{j}$ no bloco hi, $\mu$ é a média geral, $\beta_{h i}$ é o efeito do bloco hi, $\tau_{j}$ o efeito direto do tratamento $\underline{j}, \rho_{\ell(h, i, j)}$ o efeito residual do tratamento anterior ao tratamento $\mathfrak{j}$ que aparece no bloco hi, e os valores de $\varepsilon_{h i j}$ são erros aleatórios independentes, com distribuição normal de média zero e variância $\sigma^{2}$. 
Usando os métodos para o estudo de modelos lineares, determinam-se:

1 - o sistema de equações normais;

2 - os estimadores dos parâmetros:

3 - o esquema de análise de variância;

4 - a variância da estimativa da diferença entre dois efeitos diretos de tratamentos;

5 - a matriz de variâncias e covariâncias dos efeitos residuais:

6 - a variância média da estimativa da diferença entre dois efeitos residuais.

Convém ressaltar que não havendo efeitos de blocos, os resultados simplificam-se bastante e assemelham-se aos do delinea mento em blocos ao acaso, aparecendo efeitos residuais em lugar de blocos. 
3.

\section{INTRODUÇÃO}

No campo experimental, pode ser necessärio usar somente uma unidade experimental, na qual diversos tratamentos são aplicados, em períodos sucessivos; como consequência, uma observação, referente a um certo período, pode ter não só um efeito direto do tratamento aplicado no período como também um efeito residual do tratamento aplicado no período anterior.

Tal situação pode ser estudada através das Sequências Equilibradas em Série, introduzidas por FINNEY e OUTHWAITE (1955), nas quais os tratamentos são dispostos em uma série de blocos completos, de modo que o efeito residual de qualquer tratamento ocorra o mesmo número de vezes junto com o efeito direto de cada tratamento, incluindo ele mesmo (sequências de tipo I), ou de cada outro tratamento (sequências de tipo II)。 
As Sequências Equilibradas em Série são definidas adequadamente por SAMPFORD (1957), como se segue:

Uma Sequência de Tipo I de ordem $\underline{t}$ e índice $\underline{k}$ é uma cadeia fechada de letras, tal que:

1) cada uma das $t$ diferentes letras ocorre $k t$ vezes na sequência;

2) a sequência aparece em kt blocos, cada um com as $t$ diferentes letras, uma vez cada;

3) os possíveis $\underline{t}^{2}$ diferentes pares de letras ocorrem $k$ vezes cada um entre os $\mathrm{kt}^{2}$ pares de letras consecutivas na sequên cia.

Uma Sequência de Tipo II é semelhantemente definida. com a diferença de que a cadeia de letras contém somente $\underline{k(t-1)}$ blo cos e que os $\underline{t(t-1)}$ possíveis pares de diferentes letras ocorrem $\underline{k}$ vezes cada um, e uma letra não é seguida por ela mesma.

\section{Exemplos de Sequências de Tipo 1:}

$$
\text { 19) } t=6 \text { e } k=1
$$

$\begin{array}{llllll}(. & \cdot & 0 & 0 & \circ & A] \\ A & F & B & E & C & D \\ D & F & C & E & A & B \\ B & A & C & F & D & E \\ E & B & F & A & D & C \\ C & B & D & A & E & F \\ F & E & D & B & C & A\end{array}$


28) $t=4$ e $k=2$

$\begin{array}{llll}C & \cdot & \cdot & A \text { ) } \\ A & D & B & C \\ C & D & A & B \\ B & A & C & D \\ D & B & C & A \\ A & C & B & D \\ D & C & A & B \\ B & A & D & C \\ C & B & D & A\end{array}$

Exemplos de Sequências de Tipo II:

I8) $\mathrm{t}=4$ \& $k=1$

$$
\begin{array}{llll}
(0 & 0 & \cdot & A) \\
B & C & D & A \\
C & B & A & D \\
B & D & C & A
\end{array}
$$

29) $t=3$ e $k=3$

$$
\begin{array}{lll}
\text { (. } & \text {. } & \text { C) } \\
A & B & C \\
B & C & A \\
C & A & B \\
A & C & B \\
C & B & A \\
B & A & C
\end{array}
$$

Na utilização das Sequências Equilibradas em Série. como delineamentos experimentais, as linhas de uma sequência repre- 
sentam blocos completos. Se os tratamentos forem distribuidos em pe. ríodos sucessivos de acordo com as letras da sequência, o efeito di reto de qualquer tratamento ocorre simultaneamente $k$ vezes com 0 efeito residual de cada tratamento (tipo I), ou com o efeito residual de cada um dos outros tratamentos (tipo II); exceto que o efei to direto do primeiro tratamento na sequência completa ocorre somen te $(k-1)$ vezes com o efeito residual do último. Para corrigir isto, o último tratamento é também aplicado no começo da sequência como um tratamento preliminar, omitindo-se da análise qualquer observação feita nessa ocasião (SAMPFORD, 1957).

Este trabalho pretende apresentar o método geral de análise para a classe de Sequências, Equilibradas em Série, de Tipo I, consideradas por SINHA (1971), visando a preencher lacunas existentes na literatura pertinente, tais como fórmulas e deduções matemāticas. 


\section{REVISÃO DE LITERATURA}

As Sequências Equilibradas em Série foram introduzidas por FINNEY e OUTHWAITE (1955 e 1956)。Os autores abordaram as características, construção e aplicação de tais sequências, e ainda, discutiram aspectos relacionados à análise das Sequências Equilibra das em Série, de Tipo II. Entre os exemplos dados por eles, encontra-se a sequência de tipo $I, \operatorname{com} t=2$ e $k=1$,

(. A)

A $\quad B$

B A

SAMPFORD (1957), além de definir, adequadamente, as Sequências Equilibradas em Série e discutir suas propriedades gerais, descreveu, com detalhes, métodos para a construção de certas classes de sequências. Apresentou tabelas contendo as seguintes se- 
quências de tipo I:

$$
\begin{aligned}
& t=3 \text { e } k=2 \\
& \text { (.. A) } \\
& \text { A B C } \\
& \text { C A B } \\
& \text { B } C A \\
& \text { A } C \text { C } \\
& \text { B A C } \\
& \text { C B A s } \\
& t=4 \text { e } k=2 \\
& \text { (. } \therefore A \text { ) } \\
& \begin{array}{llll}
A & B & D & C
\end{array} \\
& \text { C } A \quad D \quad B \\
& \begin{array}{llll}
B & C & A & D
\end{array} \\
& D \text { C } B \text { A } \\
& \begin{array}{llll}
A & B & C & D
\end{array} \\
& \begin{array}{llll}
D & A & C & B
\end{array} \\
& \text { B D } A \text { C } \\
& C D B A \text {, }
\end{aligned}
$$


9.

$$
\begin{aligned}
& t=5 \text { e } k=2 \\
& \text { (. . . A } \\
& \begin{array}{lllll}
A & B & E & C & D
\end{array} \\
& D \text { D } \quad C \quad A \quad B \\
& \begin{array}{lllll}
B & C & A & D & E
\end{array} \\
& \text { E } A \quad D \quad B \quad C \\
& C \quad D \quad B \quad E \quad A \\
& \begin{array}{lllll}
A & E & B & D & C
\end{array} \\
& \text { C } B \text { D } A \text { A } \\
& \begin{array}{lllll}
E & D & A & C & B
\end{array} \\
& \begin{array}{lllll}
B & A & C & E & D
\end{array} \\
& D \text { C } E \text { B A, } \\
& t=6 \quad \& \quad k=1 \\
& \text { (.०००.A) } \\
& \text { A } F \text { B } E \text { C } D
\end{aligned}
$$

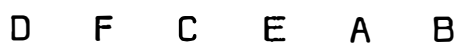

$$
\begin{aligned}
& \text { B } A C A C F \quad D \quad E \\
& \begin{array}{llllll}
E & B & F & A & D & C
\end{array} \\
& C \quad B \quad D \quad A \quad E \quad F \\
& F \quad D \quad C A \text { 。 } \\
& t=7 \text { e } k=1
\end{aligned}
$$

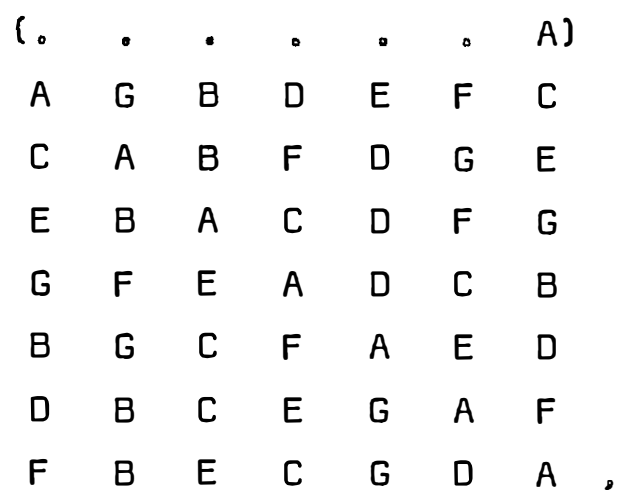




\begin{tabular}{|c|c|c|c|c|c|c|c|c|c|c|}
\hline & & . & . & . & & & 。 & 。 & \multicolumn{2}{|c|}{ A) } \\
\hline & & $H$ & $B$ & $\mathbf{G}$ & 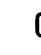 & & $=$ & $D$ & \multicolumn{2}{|l|}{$E$} \\
\hline & & C & $G$ & $D$ & $F$ & & -1 & $A$ & \multicolumn{2}{|c|}{$B$} \\
\hline & & $A$ & C & $H$ & L & & $G$ & $E$ & \multicolumn{2}{|c|}{$F$} \\
\hline & & $B$ & $E$ & $G$ & 1 & & D & $\mathrm{H}$ & \multicolumn{2}{|c|}{ C } \\
\hline & & $B$ & $D$ & $A$ & $E$ & & -1 & $F$ & \multicolumn{2}{|c|}{$G$} \\
\hline & & $B$ & $\mathrm{H}$ & $E$ & $f$ & & $=$ & C & \multicolumn{2}{|c|}{$D$} \\
\hline & & C & $E$ & $B$ & $\mathrm{~F}$ & & $A$ & $G$ & \multicolumn{2}{|c|}{$\mathrm{H}$} \\
\hline & & $\mathbf{G}$ & $F$ & $E$ & [ & & 8 & C & \multicolumn{2}{|l|}{$A$} \\
\hline & & & $t=$ & 9 & $e$ & $k=$ & 1 & & & \\
\hline c。 & . & 。 & 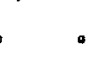 & & . & . & . & 。 & A & ) \\
\hline$A$ & I & $E$ & $D$ & $F$ & $F$ & $C$ & $\mathrm{H}$ & $G$ & E & 3 \\
\hline$B$ & G & $F$ & $\mathrm{H}$ & [ & D & $E$ & $I$ & $A$ & 0 & 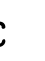 \\
\hline$C$ & $I$ & $F$ & $E$ & 0 & $G$ & $\mathrm{H}$ & $B$ & $A$ & C & 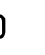 \\
\hline$D$ & $H$ & $F$ & $G$ & 1 & I & $\mathrm{B}$ & $C$ & $A$ & $E$ & $E$ \\
\hline$E$ & $H$ & $I$ & $G$ & 0 & $C$ & $B$ & $D$ & $A$ & $F$ & $=$ \\
\hline$F$ & I & $H$ & C & [ & D & B & $E$ & $A$ & $E$ & 3 \\
\hline$G$ & $D$ & $I$ & C & $E$ & $E$ & $B$ & $F$ & A & $r$ & 1 \\
\hline$H$ & $E$ & $F$ & $D$ & 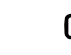 & $c$ & $\mathbf{G}$ & $A$ & $B$ & $I$ & {[} \\
\hline$I$ & $D$ & G & $E$ & $c$ & $C$ & $F$ & $B$ & $H$ & $A$ & $A$ \\
\hline
\end{tabular}




$$
t=10 \text { e } k=1
$$

\begin{tabular}{|c|c|c|c|c|c|c|c|c|c|}
\hline & . &. & . & a & 。 & . & . & . & A) \\
\hline$A$ & $\mathrm{~J}$ & $B$ & $I$ & C & $\mathrm{H}$ & 0 & $G$ & $E$ & $F$ \\
\hline$F$ & $\mathrm{~J}$ & $C$ & $I$ & $D$ & $\mathrm{H}$ & $E$ & $G$ & $A$ & $B$ \\
\hline$B$ & $A$ & C & $\mathrm{J}$ & $D$ & $I$ & $E$ & $\mathrm{H}$ & $F$ & $G$ \\
\hline$G$ & $D$ & $B$ & $\mathrm{~J}$ & $E$ & $I$ & $A$ & $F$ & $H$ & C \\
\hline C & $B$ & $D$ & $A$ & $\mathrm{E}$ & $\mathrm{J}$ & $F$ & $I$ & $G$ & $\mathrm{H}$ \\
\hline $\mathrm{H}$ & $\mathrm{J}$ & $G$ & $I$ & $F$ & $B$ & $E$ & C & $A$ & 0 \\
\hline D & C & $E$ & $B$ & $F$ & A & $G$ & $\mathrm{~J}$ & $H$ & $I$ \\
\hline$I$ & $B$ & C & $D$ & $\mathrm{~J}$ & $A$ & $\mathrm{H}$ & $G$ & $F$ & $E$ \\
\hline$E$ & $D$ & $F$ & C & $G$ & $B$ & $\mathrm{H}$ & $A$ & $I$ & $\mathrm{~J}$ \\
\hline $\mathrm{J}$ & $I$ & $H$ & $B$ & G & C & $\mathrm{F}$ & 0 & $E$ & $A$ \\
\hline
\end{tabular}

Segundo o citado autor, não existem sequências de ti po I para $t=3,4$ e 5 , no caso de $k=1$, concordando com FINNEY $e$ OUTHWAITE (1956)。 Isto pode ser verificado através da enumeração de todos os casos possíveis.

Quanto à análise estatística das sequências de tipo I, o autor parte do modelo correspondente às sequências com $k=1$,

$$
y_{i j}=\mu+\beta_{i}+\tau_{j}+\rho_{k}+\varepsilon_{i j},
$$

onde: $y_{i j}$ é a observação referente ao tratamento $\underline{j}$ no bloco $\underline{i}$;

$\mu$ é a média geral:

$\beta_{i}$ é o efeito do bloco $\underline{i}$

$\tau_{j}$ é o efeito do tratamento $\underline{j_{j}}$

$\rho_{k} e^{-}$o efeito residual do tratamento anterior ao tratamento $\underline{j}$ que aparece no bloco $\underline{i}$ 

$\varepsilon_{1 \jmath}$ são os desvios não correlacionados, com distribuição nor- mal de média zero e variância $\sigma^{2}$.

Admitindo as hipóteses $\sum_{i} \hat{\beta}_{i}=\sum_{j} \hat{\tau}_{j}=\sum_{j} \hat{\rho}_{j}=0(i, j=1, \ldots, t)$, ele estabelece as equações normaịs e indica, de maneira sucinta, um mëtodo de análise para este tipo de sequência.

Para estimar os efeitos residuais, é necessário inverter a matriz circulante e simétrica

$$
\left[\begin{array}{ccccccc}
t^{2}-2 & 1 & 0 & \cdots & 0 & 0 & 1 \\
1 & t^{2}-2 & 1 & \cdots & 0 & 0 & 0 \\
0 & 1 & t^{2}-2 & \cdots & 0 & 0 & 0 \\
\cdots & \cdots & \cdots & \cdots & \cdots & \cdots & \cdots \\
0 & 0 & 0 & \cdots & 1 & t^{2}-2 & 1 \\
1 & 0 & 0 & \cdots & 0 & 1 & t^{2}-2
\end{array}\right]
$$

de ordem txt. 0 autor apresenta um método de inversão bastante simples, com base nas propriedades da referida matriz, e, também, tabelas com a 1\% linha da inversa, para alguns casos.

0 mesmo autor, sem deduzir, apresenta as equações pa ra efeitos residuais com efeitos de blocos eliminados, no caso de $k=2$, e uma expressão para calcular a variància média da estimativa da diferença entre dois efeitos residuais para o caso de $k=1$ ou $k=2$. Ademais, a análise das sequências de tipo II foi também 
discutida brevemente.

SINHA (1971), ao pesquisar as propriedades das Sequências Equilibradas em Série, de Tipo I, considerou que os $\mathrm{kt}$ blo cos da sequência são divididos em $\underline{k}$ conjuntos de $\underline{t}$ blocos cada um. Indicando por $j 1, j 2, \ldots, j t$ os $t$ blocos do $j$-ésimo conjunto, ele admitiu que $\forall j, I \leq j \leq k$, os blocos $j 1, j 2, \ldots$ jt seguem uma das estruturas seguintes:

\section{a) Estrutura $S_{1}$}

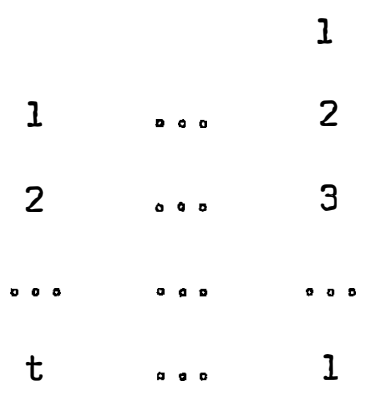

Os blocos $j 1, j 2, \ldots . . j t$ começam com os tratamentos $1,2, \ldots, t$ e terminam com $2,3, \ldots, t, 1$, respectivamente. Assim, no bloco ji, o efeito residual do tratamento $\underline{i}$ ocorre duas vezes, o do tratamento $\underline{i+1}$ não ocorre e o efeito residual de cada um dos demais tratamentos ocorre somente uma vez. 
b) Estrutura $\mathrm{S}_{2}$

$\begin{array}{ccc}1 & \ldots & t \\ t & \ldots & t-1 \\ 3 & \ldots & 2 \\ 2 & \ldots & 1\end{array}$

Os blocos $j 1, j 2, \ldots . . j$ t começam com os tratamentos $1, t, t-1, \ldots ., 3,2$ e terminam com $t, t-1, \ldots, 2,1$, respectivaren te. Assim, no bloco ji o efeito residual do tratamento $t-i+2$ (mód.t) ocorre duas vezes, o do tratamento $t-i+1$ (mód。t) não ocorre e o efei to residual de cada um dos demais tratamentos ocorre somente uma vez. Observa-se que os exemplos de Sequências Equilibradas em Série, de Tipo I, encontrados na literatura pesquisada, estão de acordo com as estruturas, consideradas por SINHA (1971).Além disso, a utilização dessas estruturas permite estabelecer uma termi nologia simples, fato observado superficialmente por SAMPFORD (1957) e mais apropriadamente por SINHA (1971)。 
15 .

\section{DESENVOLVIMENTO TEORICO}

A teoria será desenvolvida para a classe de Sequências Equilibradas em Série, de Tipo I, abordadas por SINHA (1971), na qual se podem considerar os $k t$ blocos da sequência, divididos em $\underline{k}$ conjuntos de $t$ blocos cada um e, ainda, que o h-ésimo conjunto $(\forall h, 1 \leq h \leq k)$ apresenta uma das seguintes estruturas:

\section{Estrutura $\mathrm{S}_{1}$}

\begin{tabular}{cccc} 
Blocos & & \multicolumn{3}{r}{ Tratamentos } \\
$h 1$ & 1 & $\ldots$ & 2 \\
$h 2$ & 2 & $\ldots$ & 3 \\
$\ldots$ & $\ldots$ & $\ldots$ & $\ldots$ \\
$h(t-1)$ & $t-1$ & $\ldots$ & $t$ \\
$h t$ & $t$ & $\ldots$ & 1
\end{tabular}




\section{Estrucura $\mathrm{S}_{2}$}

\begin{tabular}{cccc} 
Blocos & \multicolumn{3}{r}{ Tratamentos } \\
$h 1$ & 1 & $\ldots$ & $t$ \\
$h 2$ & $t$ & $\ldots$ & $t-1$ \\
$\ldots$ & $\ldots$ & $\ldots$ & $\ldots$ \\
$h(t-1)$ & 3 & $\ldots$ & 2 \\
$h t$ & 2 & $\ldots$ & 1
\end{tabular}

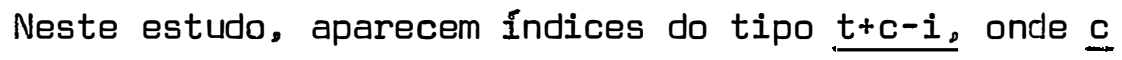
e $\underline{i}$ são inteiros, $c \geq 0$ e $i \leq t$. Se $t+c-i>t$, devem ser entendidos como $t+c-i$ (mód.t), isto é, o valor da expressão $t+c-i$ é substituido pelo resto da sua divisão por $\underline{t}$.

Os resultados obtidos neste trabalho supõem $k t>2$.

\section{1 - Anālise de Variância}

\section{1 .1 - Modelo matemático}

Considerando o modelo matemático apresentado por SAMP FORD (1957), para o caso de $k=1$, conclui-se para o caso geral(qual quer $k J$ :

$$
\begin{aligned}
y_{h i j} & =\mu+\beta_{h i}+\tau_{j}+\rho_{\ell(h, i, j)}+\varepsilon_{h i j} \ldots \ldots(4.1 .1 . a), \\
\operatorname{com} i, j & =1, \ldots, t \\
h & =1, \ldots, k
\end{aligned}
$$


onde: $y_{h i j}=$ observação referente ao tratamento $\underline{j}$ no bloco hi;

$\mu=$ média geral;

$\beta_{h i}=$ efeito do bloco hi:

$\tau_{j}=$ efeito direto do tratamento $\underline{\underline{j}}$

$\rho_{\ell(h, i, j)}=$ efeito residual do tratamento anterior ao tratamento $\underline{j}$ que aparece no bloco hi;

$\varepsilon_{\text {hij }}=$ erros aleatórios independentes, com distribuição normal de média zero e variância $\sigma^{2}$.

4.1 .2 - Sistema de equações normais

Utilizando o método dos quadrados mínimos, chega-se

facilmente ao sistema:

$\left\{\begin{aligned} \sum_{h, i, j} y_{h i j} & =\sum_{h, i, j} \hat{\mu}+\sum_{h, i, j} \hat{\beta}_{h i}+\sum_{h, i, j} \hat{\tau}_{j}+\sum_{h, i, j} \hat{\rho}_{\ell(h, i, j)} \\ \sum_{j} y_{h i j} & =\sum_{j} \hat{\mu}+\sum_{j} \hat{\beta}_{h i}+\sum_{j} \hat{\tau}_{j}+\sum_{j} \hat{\rho}_{\ell(h, i, j)} \\ \sum_{h, i} y_{h i j} & =\sum_{h, i} \hat{\mu}+\sum_{h, i} \hat{\beta}_{h i}+\sum_{h, i} \hat{\tau}_{j}+\sum_{h, i} \hat{\rho}_{\ell(h, i, j)} \\ \sum_{h, i, j / l} y_{h i j} & =\sum_{h, i, j / l} \hat{\mu}+\sum_{h, i, j / \ell} \hat{\beta}_{h i}+\sum_{h, i, j / l} \hat{\tau}_{j}+\sum_{h, i, j / l} \hat{\rho}_{\ell(h, i, j)}\end{aligned}\right.$

onde $\sum_{h, i, j / \ell}$ indica a soma das observações que incluem o efeito.resi dual do tratamento $\underline{\ell}$. 
considerando que:

$$
\text { Admitindo as restrições } \underset{h, i}{ } \bar{\beta}_{h i}=\sum_{j} \bar{\tau}_{j}=\sum_{j} \hat{\rho}_{j}=0 \text { e }
$$

$B_{h i}$ é o total das $\underline{t}$ observações do bloco hi:

$T_{j}$ é o total das kt. observações do tratamento $\underline{j}$

$R_{i}$ é o total das kt observações seguintes ao tratamento $\underline{i}$;

$G$ ë o total das $\mathrm{kt}^{2}$ observações;

tem-se o sistema

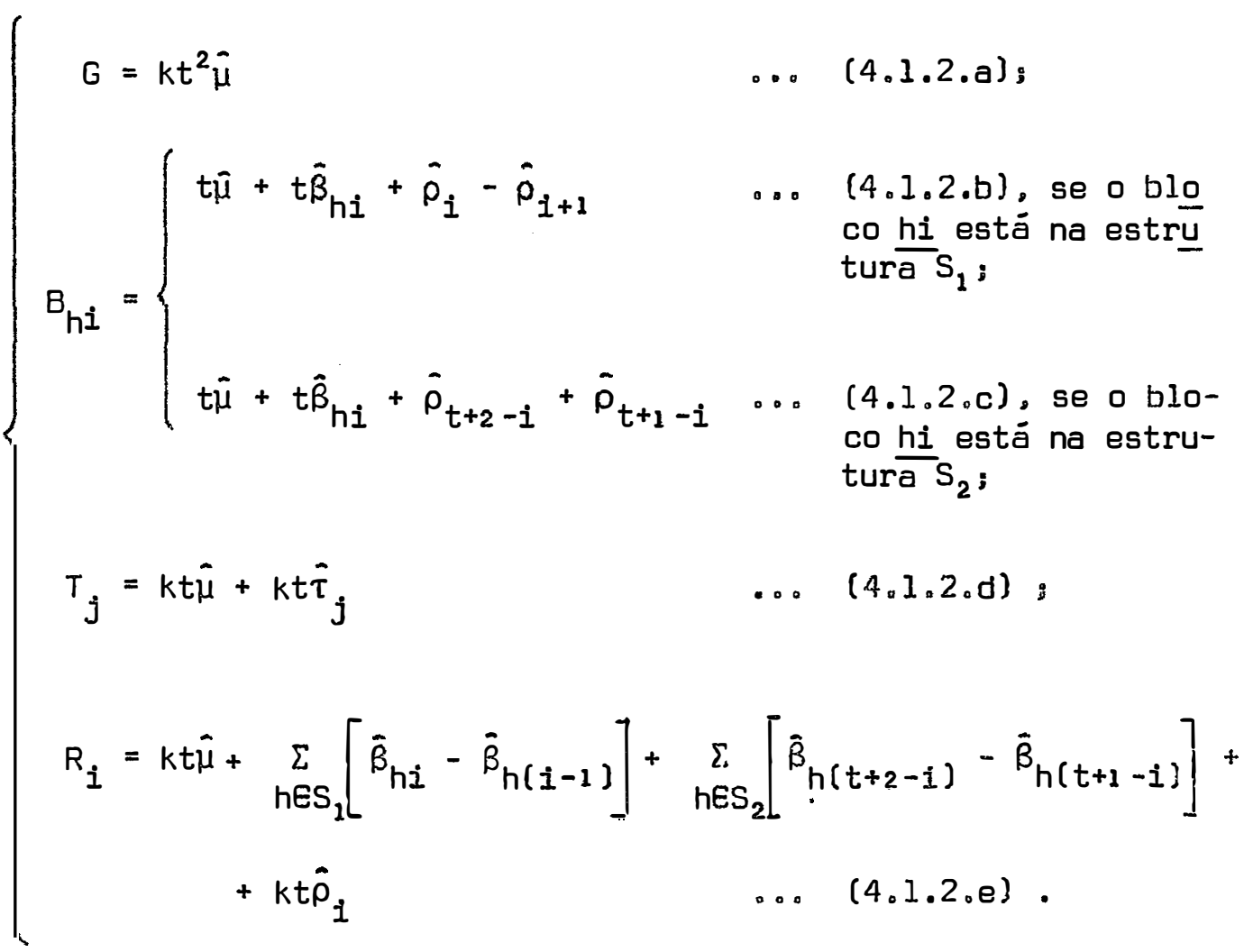




\subsection{3 - Estimadores dos parâmetros}

Da expressão $(4.1 .2 . a)$, obtẻm-se

$$
\hat{\mu}=\frac{G}{k t^{2}} \quad \ldots \quad(4.1 .3 . a) .
$$

Da expressão $(4.1 .2 . d\}$, obtêm-se

$$
\bar{\tau}_{j}=\frac{T_{j}}{k t}-\frac{G}{k t^{2}} \quad \ldots \quad \quad(4.1 .3 .6)
$$

Das expressões $(4,1.2, b)$ e $(4,1,2, c)$, obtêm-se

$$
\begin{aligned}
& \hat{B}_{h i}=\frac{B_{h i}}{t}-\frac{G}{k t^{2}}-\frac{1}{t} \cdot\left(\hat{\rho}_{i}-\hat{\rho}_{i+1}\right) \ldots \begin{array}{l}
(4.1 .3 . c), \text { se o blo- } \\
\text { co hi està na estru- } \\
\text { tura } S_{1} ;
\end{array} \\
& \hat{B}_{h i}=\frac{B_{h i}}{t}-\frac{G}{k t^{2}}-\frac{1}{t}\left(\hat{\rho}_{t+2-i}-\hat{\rho}_{t+1-i}\right) \ldots \begin{array}{l}
(4.1 .3 . d), \text { se o } \\
\text { bloco hi está na } \\
\text { estrutura } S_{2} .
\end{array}
\end{aligned}
$$

Substituindo as expressões de $\hat{\beta}_{h i}$ e $\hat{\mu}$ em $(4.1 .2 . e)$,

tem-se:

$$
R_{i}=k t \frac{G}{k t^{2}}+\sum_{h \in S_{1}}+\left[\begin{array}{l}
\frac{B_{h i}}{t}-\frac{G}{k t^{2}}-\frac{1}{t}\left(\hat{\rho}_{i}-\hat{\rho}_{i+1}\right)-\frac{B_{h(i-1)}}{t}+\frac{G}{k t^{2}}+ \\
+\frac{1}{t}\left(\hat{\rho}_{i-1}-\hat{\rho}_{i}\right)
\end{array}\right]+
$$




$$
\begin{aligned}
& +\sum_{h \in S_{2}}\left[\begin{array}{c}
\left.\frac{B_{t+2-i}}{t}-\frac{G}{k t^{2}}-\frac{1}{t}\left(\hat{p}_{i}-\hat{\rho}_{i-1}\right)-\frac{B_{t+1-i}}{t}+\frac{G}{k t^{2}}+\right] \\
\quad+\frac{1}{t}\left(\hat{\rho}_{i+1}-\hat{\rho}_{i}\right)
\end{array}\right]+k t \hat{\rho}_{i} \\
& R_{i}=\frac{G}{t}+\sum_{h \in S_{1}}\left[\frac{\left.B_{h i}-B_{h(i-1)}-\frac{1}{t}\left(2 \hat{\rho}_{i}-\hat{\rho}_{i+1}-\hat{\rho}_{i-1}\right)\right]+}{t}\right] \\
& +\sum_{h \in S_{2}}\left[\frac{\left.B_{h(t+2-i)}-B_{h(t+1-i)}-\frac{1}{t}\left(2 \hat{\rho}_{i}-\hat{\rho}_{i+1}-\hat{\rho}_{i-1}\right)\right]+}{\quad}\right. \\
& +k t \hat{\rho}_{i}
\end{aligned}
$$

\section{Então,}

$$
\begin{aligned}
k\left[\dot{\rho}_{i-1}+\left(t^{2}-2\right) \dot{\rho}_{i}+\dot{\rho}_{i+1}\right]=t R_{i} & -G-\sum_{h \in S_{1}}\left[\theta_{h i}-B_{h(i-1)}\right]- \\
& -\sum_{h \in S_{2}}\left[B_{h(t+2-i)}-B_{h(t+i-i)}\right]
\end{aligned}
$$

Indicando o segundo membro da igualdade acima por $Q_{i}$,

vem

$$
k\left[\hat{\rho}_{i-1}+\left(t^{2}-2\right) \hat{\rho}_{i}+\dot{\rho}_{i+2}\right]=Q_{i} \ldots(4.1 .3 . f)
$$

Portanto, as estimativas dos efeitos residuais são ob tidas através da resolução do sistema de equações para efeitos resí 
duais com efeitos de blocos eliminados, dado por $(4.1 .3 . f)$.

Na forma matricial, a expressão $(4.1 .3 . f)$ fica
$L \hat{\rho}=Q$
$\ldots \quad(4.1 .3 . g)$

onde:

$L=k\left[\begin{array}{ccccccc}t^{2}-2 & 1 & 0 & \ldots & 0 & 0 & 1 \\ 1 & t^{2}-2 & 1 & \ldots & 0 & 0 & 0 \\ \ldots & \ldots & \ldots & \ldots & \cdots & \ldots & \cdots \\ 0 & 0 & 0 & \ldots & 1 & t^{2}-2 & 1 \\ 1 & 0 & 0 & \ldots & 0 & 1 & t^{2}-2\end{array}\right]$

$$
\tilde{\rho}=\left[\begin{array}{c}
\hat{\rho}_{1} \\
\hat{\rho}_{2} \\
\cdots \\
\hat{\rho}_{t}
\end{array}\right] \quad \text { e }=\left[\begin{array}{c}
Q_{1} \\
Q_{2} \\
\cdots \\
Q_{t}
\end{array}\right]
$$

L é uma matriz circulante e simétrica: será simbolizada por $k\left(\left(t^{2}-2,1,0, \ldots, 0,0,1\right)\right)$. A notação $\left(\left(a_{1}, a_{2}, \ldots, a_{t}\right)\right)$, onde $a_{1}, a_{2}, \ldots, a_{t}$ são os elementos da primeira linha da matriz, será adotada para indicar matriz circulante.

Como $\hat{\rho}=L^{-1} Q$, é necessärio determinar $L^{-1}$, Isto $p \underline{\underline{O}}$ de ser feito pelo método apresentado por SAMPFORD (1957), ou pelo método mais complexo apresentado por 6000 (1950). 
Para estimar os parämetros, leva-se em conta que

$$
\hat{\beta}_{h 0} \equiv \bar{\beta}_{h t}, \dot{\rho}_{t+1} \equiv \hat{\rho}_{1} \text { e } \dot{\rho}_{0} \equiv \dot{\rho}_{t} .
$$

devido às características do delineamento, como foi observado por SAMPFORD (1957)。

\subsection{4 - Esquema de anālise de variância}

Com o procedimento usual para determinar Somas de Qua drados, obtêm-se:

a) Soma de Quadrados de Tratamentos

$$
\text { SQT }=\sum_{j=1}^{t} \hat{\tau}_{j} T_{j}=\sum_{j=1}^{t} \frac{T_{j}^{2}}{k t}-\frac{G^{2}}{k t^{2}} \quad \ldots \quad \text { (4.1.4.a)。 }
$$

b) Soma de Quadrados Total

$$
\text { SQ Total }=\sum_{h, i, j} y_{h i j}^{2}-\frac{G^{2}}{k t^{2}} \quad \ldots \quad(4.1 .4 . b) \text {. }
$$

c) Soma de Quadrados de Blocos mais Efeitos Residuais

$$
S Q(B+E R)=\sum_{i} \hat{\rho}_{i} R_{i}+\sum_{h, i} \hat{\beta}_{h i} B_{h i} \ldots(4.1 .4 . c) 。 \quad \text {. }
$$

d) Soma de Quadrados de Blocos, ignorando os Efeitos Residuais

$$
\text { SQB não } a j_{0}=\sum_{h, i} \frac{B_{h i}^{2}}{t}-\frac{G}{k t^{2}} \quad \ldots \quad(4.1 .4 . d) \text {. }
$$


e) Soma de Quadrados de Efeitos Residuais ajustados

$$
\text { SQERaj。 }=S Q(B+E R)-S Q B \text { não } a j 。 \quad \ldots \quad(4.1 .4 . e) \text { 。 }
$$

f) Soma de Quadrados dos Erros

$$
S Q E=S Q T \text { Total }-S Q(B+E R)-S Q T \quad \ldots 0 \quad(4.1 .4 . f) 。
$$

Portanto, o esquema de análise de variância é:

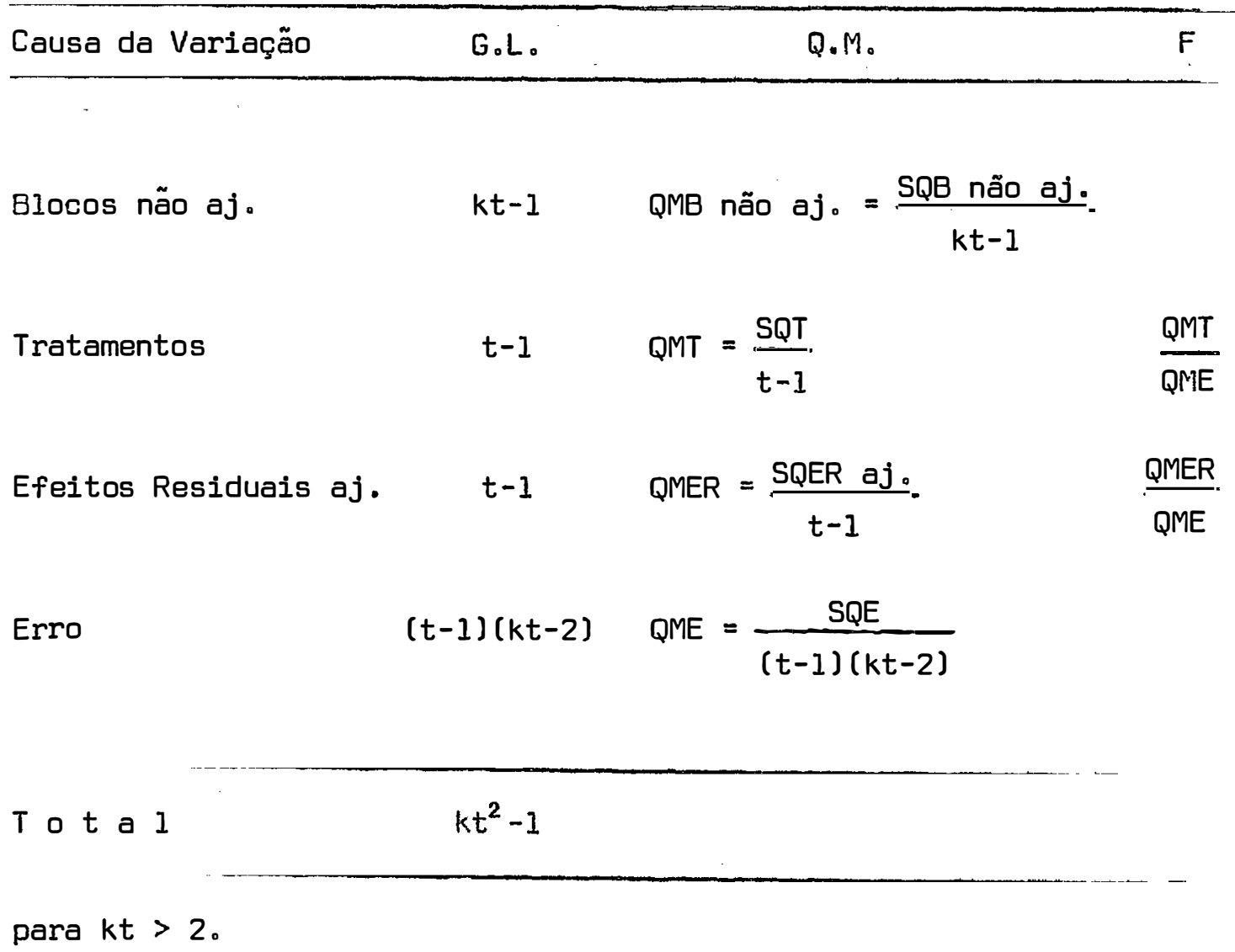




\section{2 - Variâncias das Estimativas da Diferença Entre Dois Efei- tos}

4.2.1 - Variância da estimativa da diferença entre dois efe tos diretos de tratamentos

$$
\begin{aligned}
& V\left(\hat{\tau}_{j}-\hat{\tau}_{j^{\prime}}\right)=V\left(\frac{T}{k t}-\frac{G}{k t^{2}}-\frac{T j^{\prime}}{k t}+\frac{G}{k t^{2}}\right) \\
& =\frac{l}{k^{2} t^{2}} V\left(T_{j}-T_{j}{ }^{\prime}\right) \\
& =\frac{2 \sigma^{2}}{k t} \\
& \therefore \quad[4.2 .1 .0\}
\end{aligned}
$$

\subsection{2 - Variância da estimativa da diferença entre dois efe tos residuais de tratamentos}

Geralmente, a variância da estimativa da diferença entre dois efeitos residuais depende das posições dos trat amentos en volvidos. Dar, não ser possível estabelecer uma fórmula geral.

Para um caso particular, pode-se utilizar a expressão

$$
V\left(\hat{\rho}_{i}-\hat{\rho}_{i},\right)=V\left(\hat{\rho}_{i}\right)+V\left(\hat{p}_{i},\right]-2 \operatorname{CoV}\left(\hat{\rho}_{i}, \hat{\rho}_{1},\right],
$$

sendo $V\left(\hat{\rho}_{i}\right], V\left(\hat{\rho}_{i},\right]$ e $\operatorname{CoV}\left(\hat{\rho}_{i}, \hat{\rho}_{i},\right)$ obtidas na matriz de dispersão 


$$
D(\hat{\rho})=\left[\begin{array}{cccc}
V\left(\hat{\rho}_{1}\right) & \operatorname{cov}\left(\hat{\rho}_{1}, \hat{\rho}_{2}\right) & \ldots & \operatorname{cov}\left(\hat{\rho}_{1}, \hat{\rho}_{t}\right) \\
\operatorname{cov}\left(\hat{\rho}_{1}, \hat{\rho}_{2}\right) & V\left(\hat{\rho}_{2}\right) & \ldots & \operatorname{cov}\left(\hat{\rho}_{2}, \hat{\rho}_{t}\right) \\
\ldots & \ldots & \ldots & \cdots \\
\operatorname{cov}\left(\hat{\rho}_{1}, \hat{\rho}_{t}\right) & \operatorname{cov}\left(\hat{\rho}_{2}, \hat{\rho}_{t}\right) & \cdots & v\left(\hat{\rho}_{t}\right)
\end{array}\right]
$$

Determinação de $D(\hat{\rho})$

$$
\begin{aligned}
& \text { Por definição, } \\
& D(\hat{\rho})=E\left\{[\hat{\rho}-E(\hat{\rho})][\hat{\rho}-E(\hat{\rho})]^{\prime}\right\} \text {. } \\
& \text { Em virtude de } \hat{\rho}=L^{-1} Q \text {, tem-se } \\
& D(\hat{\rho})=E\left\{\left[L^{-1} Q-E\left(L^{-1} Q\right)\right]\left[L^{-1} Q-E\left(L^{-1} Q\right)\right] \cdot\right\} \\
& =E\left\{L^{-1}[Q-E(Q)][Q-E(Q)] \cdot\left(L^{-1}\right) \cdot \cdot^{\prime}\right\} \\
& =L^{-1} D(Q)\left(L^{-1}\right)^{\prime} \\
& \text { Como } L^{-1} \text { é simétrica, pois } L \text { é simétrica, tem-se } \\
& \left(L^{-1}\right)^{0}=L^{-1} \cdot \text { Daí, vem } \\
& D(\hat{\rho})=L^{-1} D(Q) L^{-1} \quad \ldots . \quad(4.2 .2 . a)
\end{aligned}
$$

Portanto, a determinação de $D(\hat{\rho})$ depende também da determinação de $D(Q)$, o que será feito a seguir: 


$D(Q)=\left[\begin{array}{cccc}V\left(Q_{1}\right) & \operatorname{COV}\left(Q_{1}, Q_{2}\right) & \ldots & \operatorname{COV}\left(Q_{1}, Q_{t}\right) \\ \operatorname{COV}\left(Q_{1}, Q_{2}\right) & \vee\left(Q_{2}\right) & \ldots & \operatorname{COV}\left(Q_{2}, Q_{t}\right) \\ \ldots & \cdots & \ldots & \ldots \\ \operatorname{cov}\left(Q_{1}, Q_{t}\right) & \operatorname{Cov}\left(Q_{2}, Q_{t}\right) & \ldots & v\left(Q_{t}\right)\end{array}\right]$

Obtenção de $V\left(Q_{i}\right), \operatorname{cov}\left(Q_{i}, Q_{i-1}\right)$ e $\operatorname{cov}\left(Q_{i}, Q_{i},\right), \operatorname{com}|i-i \cdot|>1$

$$
\begin{aligned}
& V\left(Q_{i}\right)=V\left[\begin{array}{c}
t R_{i}-G-\sum_{h E S_{1}} B_{h i}-\sum_{h E S_{2}} B_{h(t+2-i)}+\sum_{h \in S_{1}} B_{h(i-1)}+ \\
+\sum_{h \in S_{2}} B_{h(t+1-1)}
\end{array}\right] \\
& =t^{2} V\left(R_{i}\right)+V(G)+V\left(\sum_{h \in S_{1}} B_{h i}\right)+V\left[\sum_{h \in S_{2}} B_{h(t+2-i)}\right]+ \\
& +V\left[\sum_{h E S_{1}} B_{h(i-1)}\right]+V\left[\sum_{h E S_{2}} B_{h(t+1-i)}\right]- \\
& -2 t \operatorname{cov}\left(R_{i}, G\right)-2 t \operatorname{cov}\left[R_{i}, \sum_{h \in S_{1}} B_{h i}\right]- \\
& \left.-2 t \operatorname{cov}\left[R_{i}, \sum_{h \in S_{2}} B_{h(t+2}-i\right)\right]+ \\
& +2 t \operatorname{cov}\left[R_{i}, \sum_{h \in S_{1}} B_{h(i-1)}\right]+2 t \operatorname{cov}\left[R_{i}, \sum_{h \in S_{2}} B_{h(t+1-i)}\right]+ \\
& +2 \operatorname{COV}\left(G, \sum_{h \in S_{1}} B_{h i}\right)+2 \operatorname{COV}\left[G, \sum_{h \in S_{2}} B_{h(t+2-i)}\right]-
\end{aligned}
$$




$$
\begin{aligned}
& -2 \operatorname{COV}\left[G, \sum_{h \in S_{1}} B_{h(i-1)}\right]-2 \operatorname{COV}\left[G, \sum_{h \in S_{2}} B_{h(t+1-i)}\right]+ \\
& +2 \operatorname{COV}\left[\sum_{h \in S_{1}} \theta_{h i}, \sum_{h \in S_{2}} \theta_{h(t+2-i)}\right]-
\end{aligned}
$$

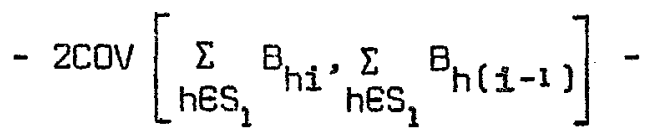

$$
\begin{aligned}
& -2 \operatorname{COV}\left[\sum_{h \in S_{1}} \theta_{h i}, \sum_{h E S_{2}} \theta_{h(t+1-i)}\right]- \\
& -2 \operatorname{COV}\left[\sum_{h \in S_{2}} B_{h(t+2-i)}, \sum_{h \in S_{1}} B_{h(i-1)}\right]- \\
& -2 \operatorname{COV}\left[\sum_{h \in S_{2}} B_{h(t+2-i)} \sum_{h \in S_{2}} B_{h(t+1-i)}\right]+ \\
& +2 \operatorname{COV}\left[\sum_{h \in S_{1}} B_{h(i-1)}, \sum_{h \in S_{2}} B_{h(t+1-i)}\right] \\
& V\left(Q_{i}\right)=k t^{3} \sigma^{2}+k t^{2} \sigma^{2}+f_{1} t \sigma^{2}+f_{2} t \sigma^{2}+f_{1} t \sigma^{2}+f_{2} t \sigma^{2}- \\
& -2 k t^{2} \sigma^{2}-4 f_{1} t \sigma^{2}-4 f_{2} t \sigma^{2}+2 f_{1} t \sigma^{2}+ \\
& +2 f_{2} t \sigma^{2}-2 f_{1} t \sigma^{2}-2 f_{2} t \sigma^{2} \text {, }
\end{aligned}
$$

onde $f_{1}$ e $f_{2}$ são as frequências das estruturas $S_{1}$ e $S_{2}$, respectivamente.

$$
\begin{aligned}
& \text { Como } f_{1}+f_{2}=k \text {, resulta } \\
& \qquad V\left(Q_{i}\right)=k t(t-2)(t+1) \sigma^{2} \quad \text {... }(4.2 .2 . b) \\
& \text { Para obter } \operatorname{cov}\left(Q_{1}, Q_{1-1}\right) \text {, observa-se que: }
\end{aligned}
$$


28.

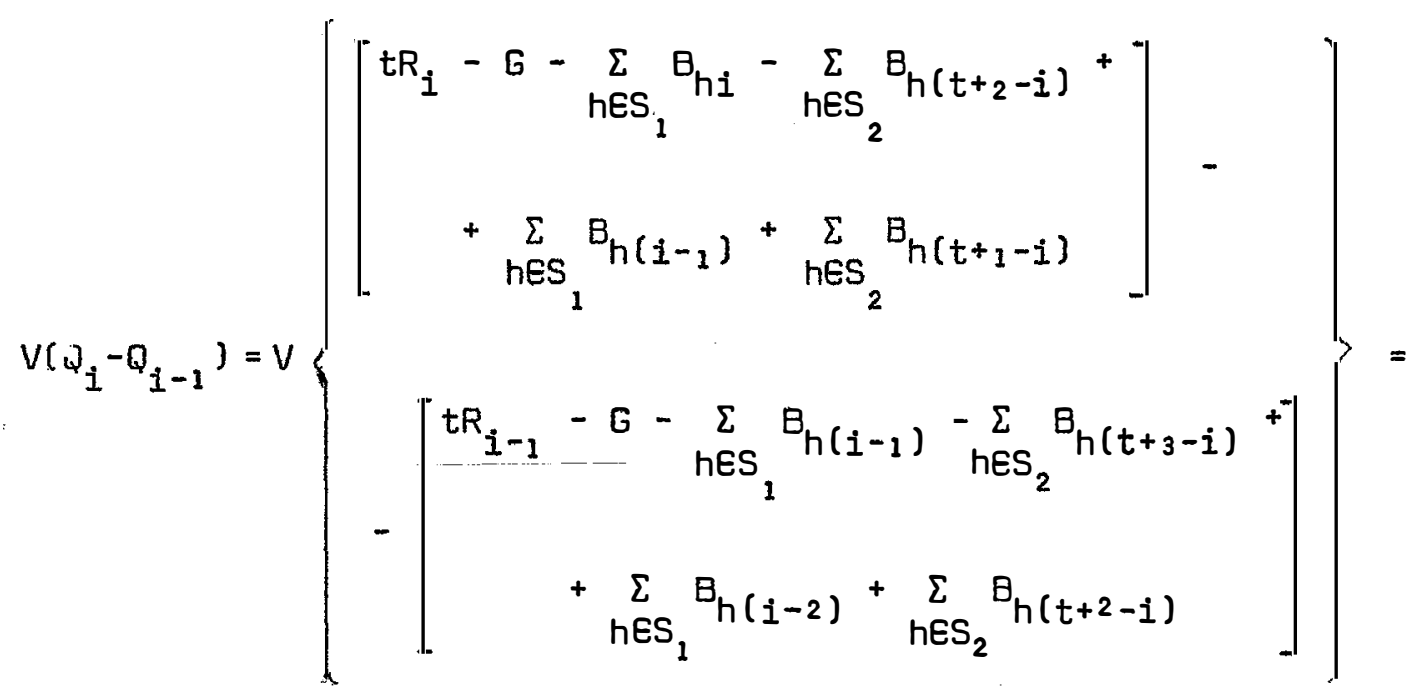

$=v\left\{\begin{aligned} t\left(R_{i}-R_{i-1}\right)+ & \sum_{h \in S_{1}}\left[B_{h(i-1)}-B_{h i}\right]+\sum_{h \in S_{2}}\left[B_{h(t+3-i)}-B_{h(t+2-i)}\right]+\cdots \\ & +\sum_{h \in S_{1}}\left[B_{h(i-1)}-B_{h(i-2)]}\right] \\ & +\sum_{h \in S_{2}}\left[B_{h(t+1-i)}-B_{h(t+2-i)}\right]\end{aligned}\right\}$

$=V\left\{\begin{aligned} t\left(R_{i}-R_{i-1}\right) & +\sum_{h \in S_{1}}\left[2 B_{h(i-1)}-B_{h i}-B_{h(i-2)}\right] t \\ & +\sum_{h \in S_{2}}\left[B_{h(t+1-i)}+B_{h(t+3-i)}-2 B_{h(t+2-i)}\right]\end{aligned}\right\}$

$$
\begin{aligned}
=V\left[t\left(R_{i}-R_{i-1}\right)\right] & +V\left\{\sum_{h \in S_{1}}\left[2 B_{h(i-1)}-B_{h i}-B_{h(i-2)}\right]\right\}+ \\
& +V\left\{\sum_{h \in S_{2}}\left[B_{h(t+1-i)}+B_{h(t+3-i)}-2 B_{h(t+2-i)}\right]\right\}+
\end{aligned}
$$


$+2 t \operatorname{cov}\left\{\left(R_{i}-R_{i-1}\right), \sum_{h \in S_{1}}\left[2 B_{h(i-1)}-B_{h i}-B_{h(i-2)}\right]\right\}+$

$+2 t \operatorname{cov}\left\{\left(R_{i}-R_{i-1}\right), \sum_{h \in S_{2}}\left[\begin{array}{l}B_{h(t+1-i)}+B_{h(t+3-i)}-1 \\ -2 B_{h(t+2-i)}\end{array}\right] \mid+\right.$

$+2 \operatorname{cov}\left\{\begin{array}{l}\sum_{h \in S_{1}}\left[2 B_{h(i-1)}-B_{h i}-B_{h(i-2)}\right], \\ \sum_{h \in S_{2}}\left[B_{h(t+1-i)}+B_{h(t+3-i)}-2 B_{h(t+2-i)}\right]\end{array}\right\}$

$V\left(Q_{1}-Q_{1-1}\right)=2 k t^{3} \sigma^{2}+6 f_{1} t \sigma^{2}+6 f_{2} t \sigma^{2}-12 f_{1} t \sigma^{2}-12 f_{2} t \sigma^{2}$.

Como $f_{1}+f_{2}=k$, resulta $V\left(Q_{i}-Q_{i-1}\right)=2 k t\left(t^{2}-3\right) \sigma^{2}$.

Portanto,

$$
\begin{aligned}
\operatorname{Cov}\left(Q_{i}, Q_{i-1}\right) & =\frac{1}{2}\left[V\left(Q_{i}\right)+V\left(Q_{i-1}\right)-V\left(Q_{i}-Q_{i-1}\right)\right] \\
& =\frac{1}{2}\left[2 k t(t-2)(t+1)-2 k t\left(t^{2}-3\right)\right] \sigma^{2} \\
& =-k t(t-1) \sigma^{2} \ldots \quad(4.2 .2 . c) .
\end{aligned}
$$

Para obter $\operatorname{cov}\left(Q_{i}, Q_{i},\right)$, com $\left|i-i^{1}\right|>1$, observa-se que: 
30.

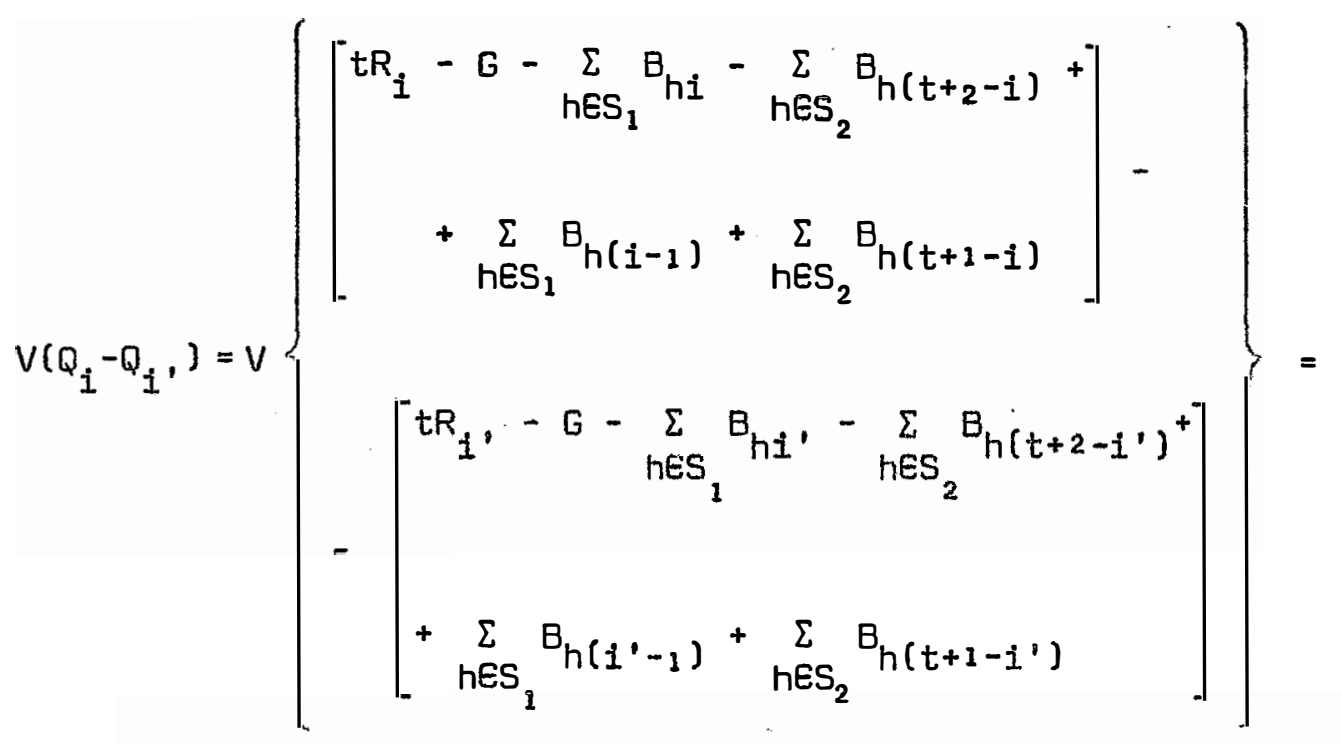

$$
\begin{aligned}
& =v\left\{\begin{aligned}
t\left(R_{i^{\prime}}-R_{i^{\prime}}\right) & +\sum_{h \in S_{1}}\left(B_{h i^{\prime}}-B_{h i^{\prime}}+\sum_{h \in S_{2}}\left[B_{h(t+2-i !)}-B_{h(t+2-i)]+}\right.\right. \\
& +\sum_{h \in S_{1}}\left[B_{h(i-1)}-B_{\left.h\left(i^{\prime}-1\right)\right]+}\right. \\
& +\sum_{h \in S_{2}}\left[B_{h(t+1-i)}-B_{h\left(t+1-i^{\prime}\right)}\right]
\end{aligned}\right.
\end{aligned}
$$

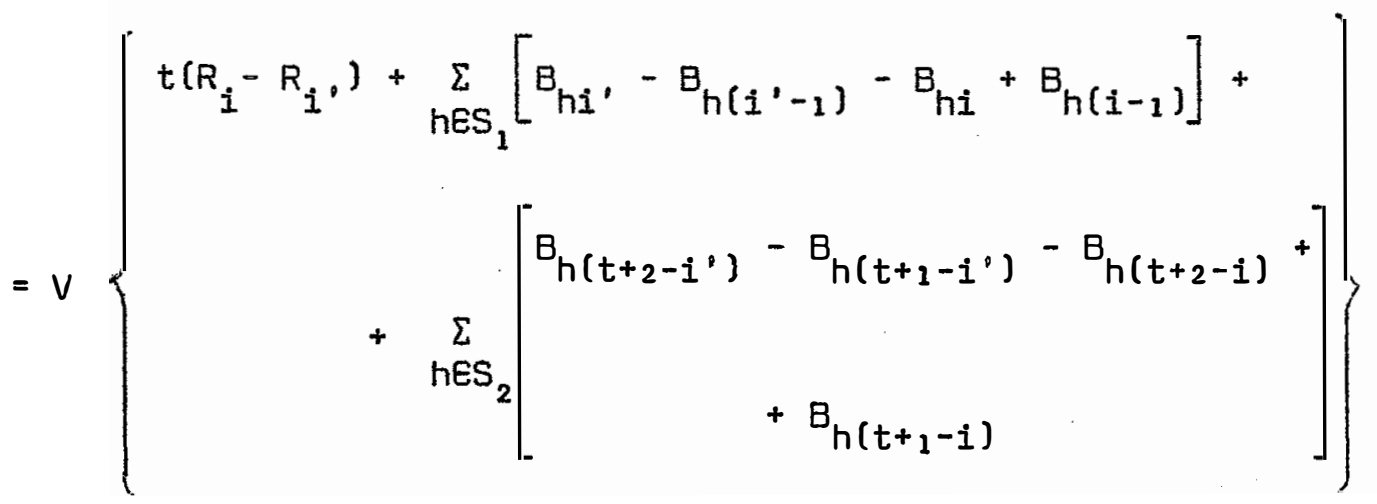


31.

$$
\begin{aligned}
& V\left(Q_{i}-Q_{i},\right)=V\left[t\left(R_{i}-R_{i},\right)\right]+V\left\{\sum_{h \in S_{1}}\left[B_{h i} \cdot-B_{h\left(i^{\prime}-1\right)}-B_{h i}+B_{h(i-1)}\right]\right\}+ \\
& \left.+V_{h \in S_{2}}\left[\begin{array}{c}
B_{h\left(t+2-i^{\prime}\right)}-B_{h\left(t+t_{1}-i^{\prime}\right)}-B_{h(t+2-i)}+ \\
+B_{h(t+1-i)}
\end{array}\right]\right\}+ \\
& +2 t \operatorname{cov}\left\{\left(R_{i}-R_{i},\right), \sum_{h \in S_{1}}\left[\begin{array}{c}
B_{h i}-B_{h(i-1)}-B_{h i}+1 \\
+B_{h(i-1)}
\end{array}\right]\right\}+
\end{aligned}
$$

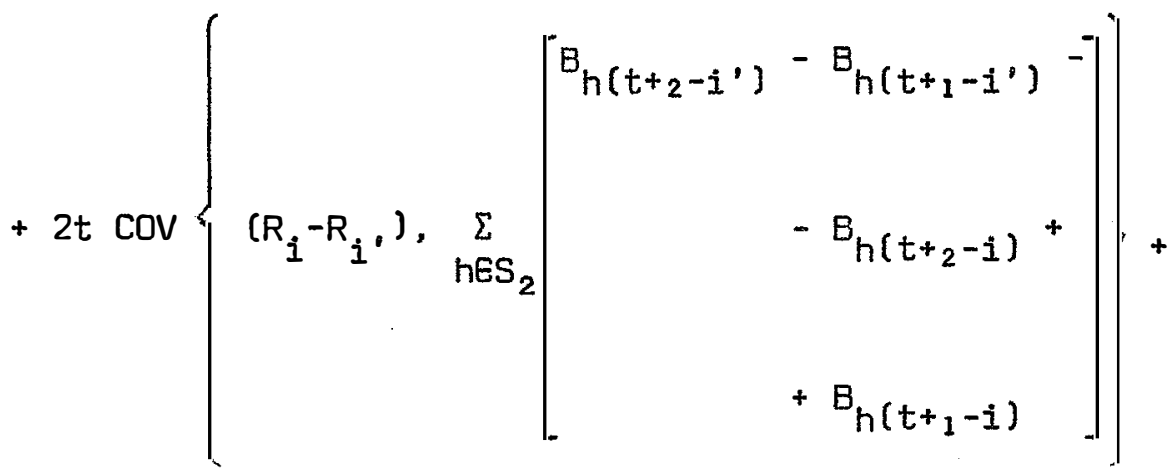

$$
\begin{aligned}
& +2 \operatorname{cov}\left\{\begin{array}{c}
\sum_{h \in S_{1}}\left[B_{h i},-B_{h\left(i^{\prime}-1\right)}-B_{h i}+B_{h(i-1)}\right] \\
\sum_{h \in S_{2}}\left[\begin{array}{c}
B_{h(t+2-i !)}-B_{h\left(t+1-i^{\prime}\right)}-B_{h(t+2-i)} \\
+B_{h(t+1-i)}
\end{array}\right]
\end{array}\right\}
\end{aligned}
$$




$$
\begin{gathered}
V\left(Q_{i}-Q_{i}\right)=2 k t^{3} \sigma^{2}+4 f_{1} t \sigma^{2}+4 f_{2} t \sigma^{2}-8 f_{1} t \sigma^{2}-8 f_{2} t \sigma^{2} . \\
\text { Como } f_{1}+f_{2}=k \text {, resulta } \\
V\left(Q_{i}-Q_{i},\right)=2 k t\left(t^{2}-2\right) \sigma^{2} .
\end{gathered}
$$

Portanto,

$$
\begin{aligned}
\operatorname{Cov}\left(Q_{i}, Q_{i},\right) & =\frac{1}{2}\left[V\left(Q_{i}\right)+V\left(Q_{i}\right)-V\left(Q_{i}-Q_{1}\right)\right] \\
& =\frac{1}{2}\left[2 k t(t-2)(t+1)-2 k t\left(t^{2}-2\right)\right] \sigma^{2} \\
& =-k t^{2} \sigma^{2}
\end{aligned}
$$

Assim sendo, vem

$$
D(Q)=k t \sigma^{2}\left[\begin{array}{cccccc}
(t-2)(t+1) & -(t-1) & -t & \ldots & -t & -(t-1) \\
-(t-1) & (t-2)(t+1) & -(t-1) & \ldots & -t & -t \\
\ldots & \ldots & \ldots & \ldots & \ldots & \ldots \\
-(t-1) & -t & -t & \ldots & -(t-1) & (t-2)(t+1)
\end{array}\right]
$$

Substituindo $(4.2 .2 . e)$ na expressão $(4.2 .2 . a)$, tem-se

$$
D(\hat{\rho})=k t \sigma^{2} L^{-1}\left(\left(t^{2}-t-2,-t+1,-t, \ldots,-t,-t+1\right)\right) L^{-1} \ldots \quad(4.2 .2 . f) 。
$$


33.

Daí, determinada a matriz $L^{-1}$ para um caso particular, obtém-se facilmente $D(\hat{\rho})$.

\subsection{3 - Variância média da estimativa da diferença entre dois efeitos residuais de tratamentos}

Pode-se tomar, para comparar dois efeitos residuais, a variância média da estimativa da diferença entre dois efeitos residuais, $\bar{V}\left(\hat{\rho}_{i}-\hat{\rho}_{i},\right)$, em lugar de $V\left(\hat{\rho}_{i^{\prime}}-\hat{\rho}_{i^{\prime}}\right)$.

Por definição, tem-se:

$$
\begin{aligned}
& \sum V\left(\hat{\rho}_{i}-\hat{\rho}_{i},\right) \\
& \nabla\left(\hat{\rho}_{i}-\hat{\rho}_{i}\right)=\frac{i \neq i^{\circ}}{t(t-1)} \quad \ldots \quad[4.2 .3 . a] \text {. } \\
& \text { Lema 1: } \quad V\left(\bar{\rho}_{i}\right)+\sum_{\substack{i^{\prime}=1 \\
i \neq i}}^{t} \operatorname{cov}\left(\bar{\rho}_{i}, \hat{\rho}_{i},\right)=0, \quad \forall i=1, \ldots, t .
\end{aligned}
$$

Demonstração:

$$
\text { Sabe-se que }
$$

$L \hat{\rho}=Q$,

isto $\dot{e}$ : 
34.

$k\left(\left(t^{2}-2,1,0, \ldots, 0,1\right)\right)\left[\begin{array}{c}\hat{\rho}_{1} \\ \hat{\rho}_{2} \\ \cdots \\ \hat{\rho}_{t}\end{array}\right]=\left[\begin{array}{c}Q_{1} \\ Q_{2} \\ \cdots \\ Q_{t}\end{array}\right]$

Por propriedade de matrizes, tem-se

$$
\begin{aligned}
& E_{1 \times t} k\left(\left(t^{2}-2,1,0 \ldots, 0,1\right)\right) \\
& \text { onde } E_{l \times t}=[1,1, \ldots, 1] .
\end{aligned}\left[\begin{array}{c}
\hat{\rho}_{1} \\
\hat{\rho}_{2} \\
\ldots \\
\hat{\rho}_{t}
\end{array}\right]=E_{1 \times t}\left[\begin{array}{c}
Q_{1} \\
Q_{2} \\
\ldots \\
Q_{t}
\end{array}\right]
$$

Então 。

$$
k t^{2} \quad E_{1 \times t}\left[\begin{array}{c}
\hat{\rho}_{1} \\
\hat{\rho}_{2} \\
\cdots \\
\hat{\rho}_{t}
\end{array}\right]=\sum_{i=1}^{t} Q_{i}
$$

ou

$$
k t^{2} \quad \sum_{i=1}^{t} \hat{\rho}_{i}=\sum_{i=1}^{t} Q_{i}
$$

Como

$$
\sum_{i}^{t} Q_{i}=\sum_{i=1}^{t}\left\{t R_{i}-G-\sum_{h \in S_{1}}\left[B_{h i}-B_{h(i-1)}\right]-\sum_{h \in S_{2}}\left[B_{h(t+2-i)}-B_{h(t+1-i)}\right]\right\}
$$




$$
\sum_{i}^{t} Q_{i}=t G-t G-G+G=0 \text {. }
$$

tem-se

$$
k t^{2} \sum_{i=1}^{t} \hat{\rho}_{i}=0
$$

logo,

$$
\sum_{i=1}^{t} \hat{\rho}_{i}=0
$$

\section{Portanto,}

$$
\operatorname{cov}\left(\hat{\rho}_{i^{\prime}}, \sum_{i^{\prime}=1}^{t} \hat{\rho}_{i^{\prime}}\right]=\operatorname{cov}\left[\hat{\rho}_{i^{\prime}}, 0\right]=0 \quad, \forall i=1 \ldots, t \quad(4.2 .3 . b)
$$

\section{Por outro lado,}

$$
\operatorname{cov}\left(\hat{\rho}_{i^{\prime}} \sum_{i^{\prime}=1}^{t} \hat{\rho}_{i^{\prime}}\right)=V\left(\hat{\rho}_{i^{\prime}}\right)+\sum_{\substack{i^{\prime}=1 \\ i \neq i^{\prime}}}^{t} \operatorname{cov}\left(\hat{\rho}_{i^{\prime}} \hat{\rho}_{i^{\prime}}\right) \quad \ldots \quad(4.2 .3 . c)
$$

Comparando $(4.2 .3 . t) \operatorname{com}(4.2 .3 . c)$, verifica-se que

$$
v\left(\hat{\rho}_{i}\right)+\sum_{\substack{i^{\prime}=1 \\ i \neq i^{\prime}}}^{t} \operatorname{cov}\left(\hat{\rho}_{i}, \hat{\rho}_{i},\right)=0, \forall i=1, \ldots, t \quad(4.2 .3 . d),
$$




$$
\text { Lema 2: } \sum_{\substack{i, i^{\circ} \\ i \neq i^{\circ}}} V\left(\hat{\rho}_{i}-\hat{\rho}_{i,}\right]=2 t \sum_{i=1}^{t} \sigma_{i 1}, \quad \text { onde } \sigma_{i i}=V\left(\hat{\rho}_{i}\right) \text {. }
$$

Demonstração:

Seja

$D(\rho)=\left[\begin{array}{llll}\sigma_{11} & \sigma_{12} & \ldots & \sigma_{1 t} \\ \sigma_{21} & \sigma_{22} & \ldots & \sigma_{2 t} \\ \ldots & \ldots & \ldots & \ldots \\ \sigma_{t 1} & \sigma_{t 2} & \ldots & \sigma_{t t}\end{array}\right]$

Pelo lema 1, tem-se

$$
\left[\begin{array}{c}
\sigma_{11}+\sigma_{12}+\ldots+\sigma_{1 t} \\
\sigma_{21}+\sigma_{22}+\ldots+\sigma_{2 t} \\
\ldots \ldots \ldots \ldots \ldots+\sigma_{t t}
\end{array}\right]=\left[\begin{array}{c}
0 \\
\sigma_{t 1}+\sigma_{t 2}+\ldots \ldots \\
0 \\
0
\end{array}\right]
$$

Sejam

$$
\begin{aligned}
& \sum_{i^{\prime}>1}^{t} V\left(\bar{\rho}_{1}-\hat{\rho}_{i^{\prime}}\right)=N_{1}, \sum_{\substack{i^{\prime}=1 \\
i^{\prime} \neq 2}}^{t} V\left(\hat{\rho}_{2}-\hat{\rho}_{1^{\prime}}\right)=N_{2}, \ldots . \\
& \sum_{j=1}^{t-1} V\left(\hat{\rho}_{t}-\hat{\rho}_{i^{\prime}}\right)=N_{t} .
\end{aligned}
$$




\section{Então.}

$$
\sum_{\substack{i, i^{\prime} \\ i \neq i^{\prime}}} V\left(\hat{p}_{i}-\hat{\rho}_{i},=N_{1}+N_{2}+\ldots+N_{t} \quad(4.2 .3 . e) .\right.
$$

Por outro lado,

$$
\begin{aligned}
N_{1}=V\left(\hat{\rho}_{1}-\hat{\rho}_{2}\right)+\ldots+V\left(\hat{\rho}_{1}-\hat{\rho}_{t}\right]=V\left(\hat{\rho}_{1}\right) & +V\left(\hat{\rho}_{2}\right)-2 \operatorname{COV}\left(\hat{\rho}_{1}, \hat{\rho}_{2}\right]+\ldots+ \\
& +V\left(\hat{\rho}_{1}\right)+V\left(\hat{\rho}_{t}\right)-2 \operatorname{cov}\left(\hat{\rho}_{1}, \hat{\rho}_{t}\right) \\
= & \sigma_{11}+\sigma_{22}-2 \sigma_{12}+\sigma_{11}+\sigma_{33}- \\
& -2 \sigma_{13}+\ldots+\sigma_{11}+\sigma_{t t}-2 \sigma_{1 t} \\
= & (t-1) \sigma_{11}+\sigma_{22}+\ldots+\sigma_{t t}- \\
& -2\left(\sigma_{12}+\ldots+\sigma_{1 t}\right),
\end{aligned}
$$

mas,

$$
-\sigma_{11}=\sigma_{12}+\ldots+\sigma_{t t} \text {, }
$$

logo,

$$
N_{1}=t \sigma_{11}+\sigma_{11}+\ldots+\sigma_{1 t} \text {. }
$$

Analogamente, obtêm-se:

$$
\begin{aligned}
& N_{2}=t \sigma_{22}+\sigma_{11}+\ldots+\sigma_{t t}: \\
& \ldots \ldots \ldots \ldots \ldots \ldots \ldots \ldots \ldots \ldots \\
& N_{t}=t \sigma_{t t}+\sigma_{11}+\ldots \ldots+\sigma_{t t}
\end{aligned}
$$


Substituindo as expressões de $N_{1}, N_{2}, \ldots, N_{t}$ em $(4.2 .3 . e)$, resulta

$$
\sum_{\substack{i, i^{\prime} \\ i \neq i^{\prime}}} V\left(\bar{\rho}_{i}-\bar{\rho}_{i},\right)=2 t \sum_{i=1}^{t} \sigma_{i i} \ldots \quad(4.2 .3 . f),
$$

Aplicendo o lema 2 na expressão $(4.2 .3 . a)$, tem-se

$$
\bar{V}\left(\hat{\rho}_{i}-\hat{\rho}_{i^{\prime}}\right)=\frac{2 \sum_{i=1} \sigma_{i i}}{t-1}
$$

Utilizando a definição de traço de matriz, segue-se

que

$$
\bar{V}\left(\hat{\rho}_{i}-\hat{\rho}_{i^{\prime}}\right)=\frac{2 \operatorname{tr} D(\hat{\rho})}{t-1} \text {. }
$$

Considerando $(4.2 .2 . a)$, pode-se escrever

$$
\bar{V}\left(\hat{\rho}_{i}-\hat{\rho}_{i},\right)=\frac{2}{t-1} \operatorname{tr}\left[L^{-1} D(Q) L^{-1}\right] .
$$

Por propriedade de traço de matriz, vem

$$
\begin{aligned}
& \bar{V}\left(\hat{\rho}_{i}-\hat{\rho}_{i},\right)=\frac{2}{t-1} \operatorname{tr}\left[L^{-1} L^{-1} D(Q)\right] \\
& \text { Como } L^{-1}=\left(L^{-1}\right) \text {, pois } L^{-1} \text { é simétrica, segue-se que } \\
& \bar{V}\left(\rho_{i}-\hat{\rho}_{i},\right)=\frac{2}{t-1} \operatorname{tr}\left[L^{-1}\left(L^{-1}\right) \cdot D(Q)\right] .
\end{aligned}
$$


Por propriedade de matriz inversa, vem

$$
\bar{V}\left(\hat{\rho}_{i}-\hat{\rho}_{i^{\prime}}\right)=\frac{2}{t-1} \operatorname{tr}\left[\left(L^{\prime} L\right)^{-1} D(Q)\right] \quad \ldots \quad(4.2 .3 . g)_{0}
$$

Desde que $L$ e L' são matrizes circulantes e simétricas, também são $L^{\prime} L$ e $\left(L^{\prime} L\right)^{-1}$, vide GODD (1950). Daí, seja

$$
(L \cdot L)^{-1}=C=\left(\left(c_{1}, c_{2}, \ldots, c_{t}\right)\right\} .
$$

Como

$$
D(Q)=k t \sigma^{2}\left(\left(t^{2}-t-2,-t+1,-t, \ldots,-t,-t+1\right)\right),
$$

segue-se que

$$
\begin{aligned}
& \operatorname{tr}\left[(L \cdot L)^{-1} D(Q)\right]=k t^{2} \sigma^{2}\left[\begin{array}{c}
c_{1}\left(t^{2}-t-2\right)-c_{2}(t-1)-c_{3} t-\ldots- \\
-c_{t-1} t-c_{t}(t-1)
\end{array}\right] \\
& =k t^{2} \sigma^{2}\left[c_{1}\left(t^{2}-2\right)+\left(c_{2}+c_{t}\right)-t \sum_{i=1}^{t} c_{i}\right]
\end{aligned}
$$

Assim sendo, tem-se

$$
\nabla\left(\hat{\rho}_{i}-\hat{\rho}_{1},\right)=\frac{2 k t^{2} \sigma^{2}\left[c_{1}\left(t^{2}-2\right)+\left(c_{2}+c_{t}\right)-t \underset{i}{\Sigma} c_{i}\right]}{t-1} \quad(4 \cdot 2 \cdot 3 . h) .
$$

Lema 3: $\sum_{i=1} c_{1}=\frac{1}{k^{2} t^{4}}$. 
40 。

Demonstração:

$$
\begin{aligned}
& \text { Sabe-se que } \\
& \qquad L=k\left(\left(t^{2}-2,1,0, \ldots, 0,1\right)\right\} . \\
& \text { Então, } \\
& L^{\prime} L E_{t \times 1}=L^{\prime}\left(k t^{2} E_{t \times 1}\right)=k t^{2} L^{\cdot} E_{t \times 1}=k^{2} t^{4} E_{t \times 1} .
\end{aligned}
$$

Portanto,

$$
\left(L^{\prime} L\right)^{-1}\left(L^{\prime} L\right) E_{t \times 1}=\left(L^{\prime} L\right)^{-1} k^{2} t^{4} E_{t \times 1} \text {. }
$$

ou ainda,

$$
I E_{t \times I}=k^{2} t^{4}[L \cdot L]^{-1} E_{t \times I} \text {. }
$$

logo,

$$
\left(L^{\prime} L\right)^{-1} E_{t \times 1}=\frac{1}{k^{2} t^{4}} E_{t \times 1}
$$

\section{Como}

$$
\left(L^{\prime} L\right)^{-1} E_{t \times 1}=\sum_{i} c_{i} E_{t \times 1},
$$

concluiu-se que

$$
\begin{gathered}
\sum_{i=1} c_{i}=\frac{1}{k^{2} t^{4}} \quad \ldots \quad(4.2 .3 . i), \\
\text { c.q.d. }
\end{gathered}
$$


41.

\section{Aplicando o lema 3 na expressão $(4.2 .3 . h)$, vem}

$$
\bar{V}\left(\hat{\rho}_{i}-\hat{\rho}_{i}{ }^{\prime}\right)=\frac{2 k t^{2}}{t-1}\left[c_{1}\left(t^{2}-2\right)+c_{2}+c_{t}-\frac{1}{k^{2} t^{3}}\right] \sigma^{2} \quad(4 \cdot 2 \cdot 3 \cdot j) .
$$

Lema 4: Se $v_{1}$ é o elemento da diagonal da matriz

$$
\left(\left(t^{2}-2,1,0, \ldots, 0,1\right)\right)^{-1}
$$

então

$$
c_{1}\left(t^{2}-2\right)+c_{2}+c_{t}=\frac{v_{1}}{k^{2}}
$$

Demons tração:

Seja

$$
L^{-1}=\frac{1}{k}\left(\left[v_{1}, v_{2}, \ldots, v_{t}\right)\right] .
$$

Como

$$
\left(L^{0} L\right)^{-1}=L^{-1}\left(L^{0}\right)^{-1}=L^{-1}\left(L^{-1}\right)^{\prime},
$$

tem-se

$$
\left(L^{\prime} L\right)^{-1}=\frac{1}{k^{2}}\left(\left(v_{1}, v_{2}, \ldots, v_{t}\right)\right\}\left(\left(v_{1}, v_{2}, \ldots, v_{t}\right)\right)^{\prime} .
$$


Daí,

$$
\begin{aligned}
& c_{1}=\frac{1}{k^{2}}\left[v_{1}, v_{2}, \ldots, v_{t}\right]\left[\begin{array}{c}
v_{1} \\
v_{2} \\
\ldots \\
v_{t}
\end{array}\right] \text {, } \\
& c_{2}=\frac{1}{k^{2}}\left[v_{1}, v_{2}, \ldots, v_{t}\right]\left[\begin{array}{c}
v_{t} \\
v_{1} \\
\ldots \\
v_{t-1}
\end{array}\right] e \\
& c_{t}=\frac{1}{k^{2}}\left[v_{1}, v_{2}, \ldots, v_{t}\right]\left[\begin{array}{c}
v_{2} \\
\cdots \\
v_{t} \\
v_{t-1}
\end{array}\right]
\end{aligned}
$$

Então, 


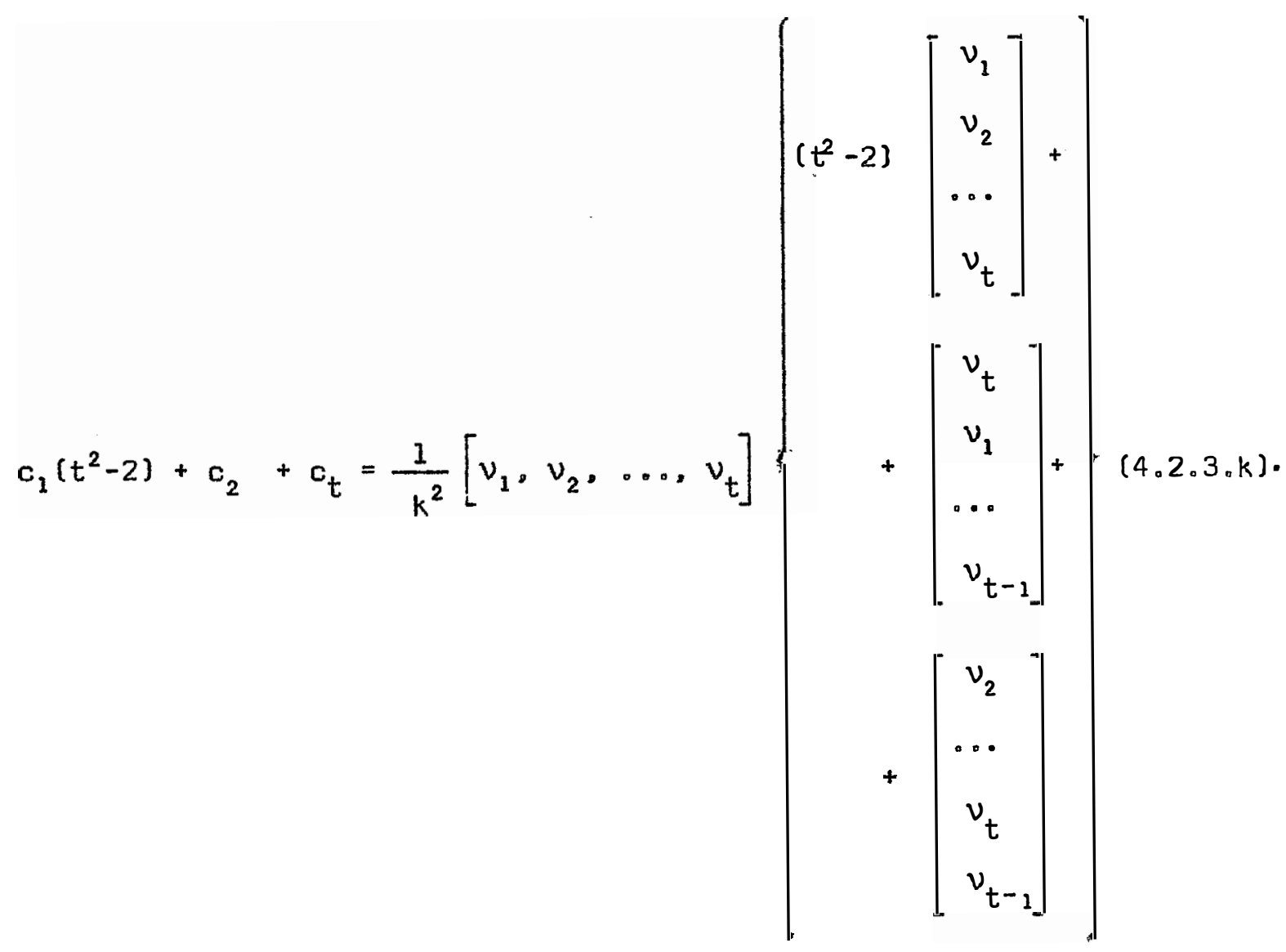

Visto que

$$
L\left(L^{-1}\right)^{\prime}=I
$$

(pois, $L L^{-1}=I$ e $\left(L^{-1}\right) \cdot=L^{-1}$ ), ou seja.

$$
k\left(\left(t^{2}-2,1,0, \ldots, 0,1\right)\right) \frac{1}{k}\left(\left(\nu_{1}, \nu_{2}, \ldots, \nu_{t}\right)^{\prime}=I_{0}\right.
$$

a expressão seguinte resulta da multiplicação da matriz $L$ pela primeira coluna da $\left(L^{-1}\right) \cdot$ : 


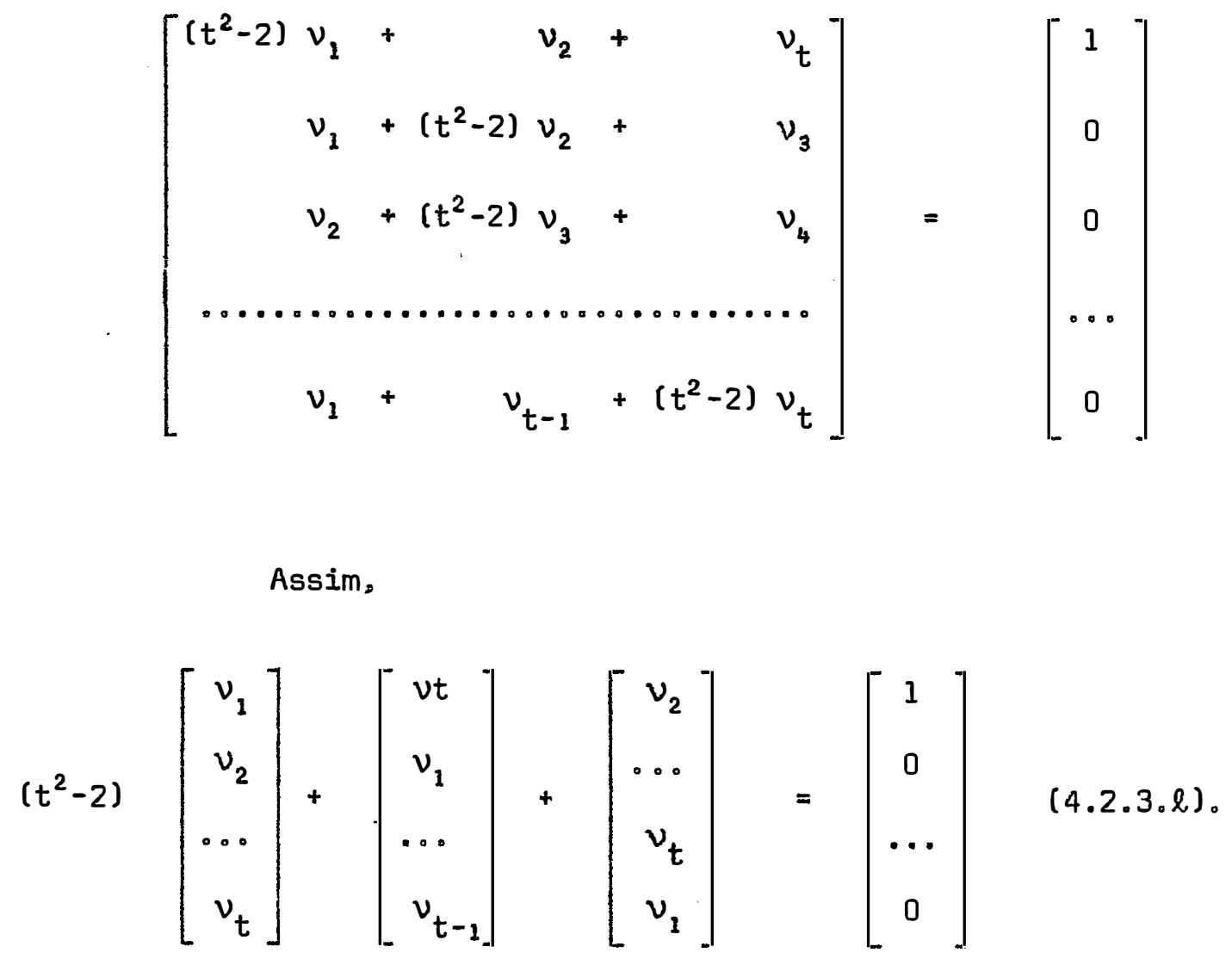

Substituindo $(4.2 .3 . l)$ em $(4.2 .3 . k)$, resulta

$$
\begin{aligned}
& c_{1}\left(t^{2}-2\right)+c_{2}+c_{t}=\frac{1}{k^{2}}\left[v_{1}, v_{2}, \ldots ., v_{t}\right]\left[\begin{array}{c}
1 \\
0 \\
\ldots \\
0
\end{array}\right] \\
& =\frac{v_{1}}{k^{2}} \quad \ldots \quad(4.2 .3 . m), \\
& \text { c.q.d. }
\end{aligned}
$$

Aplicando o lema 4 na expressão $(4.2 .3 . j)$, vem final mente 


$$
\bar{V}\left(\bar{\rho}_{i}-\hat{p}_{i},\right)=\frac{2 k t^{2}}{t-1}\left[\frac{v_{1}}{k^{2}}-\frac{1}{k^{2} t^{3}}\right] \sigma^{2},
$$

ou

$$
\bar{V}\left(\hat{\rho}_{i}-\hat{\rho}_{1},\right)=\frac{2 t^{2}}{t-1}\left[\frac{v_{1}}{k}-\frac{1}{k t^{3}}\right] \ldots(4.2 .3 . n) .
$$

\section{3 - Caso Particular}

No caso de não se considerarem efeitos de blocos,tem-se o modelo matemático

$$
y_{h i j}=\mu+\tau_{j}+p_{i}+\varepsilon_{h i j} \quad \ldots \quad \text { (4.3.a), }
$$

$$
\begin{aligned}
\operatorname{com} h & =1, \ldots, k_{i} \\
i, j & =1, \ldots, t ;
\end{aligned}
$$

onde, $y_{\text {hij }}=h$-ésima observação referente ao efeito direto do trata mento $\underline{j}$ com o efeito residual do tratamento $\underline{i}_{i}$

$\mu=$ média geral:

$\tau_{j}=$ efeito direto do tratamento $\underline{j}_{j}$

$\rho_{i}=$ efeito residual do tratamento anterior ao tratamento $\underline{j}_{3}$

$\varepsilon_{\text {hij }}=$ erros aleatórios independentes, com distribuição normal de média zero e variância $\sigma^{2}$.

Considerando o modelo $\{4.3 . a\}$, facilmente obtēm-se: 
1) Sistema de Equações Normais

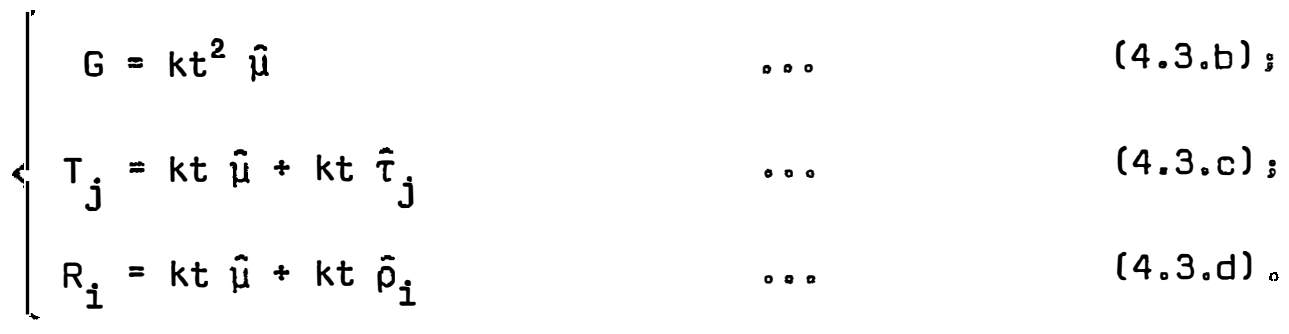

2) Estimadores dos Parâmetros

$$
\begin{array}{llll}
\bar{\mu} & =\frac{G}{k t^{2}} & \ldots & (4.3 . e) ; \\
\bar{\tau}_{j} & =\frac{T j}{k t}-\frac{G}{k t^{2}} & \ldots & (4.3 . f) \\
\tilde{p}_{i}=\frac{R_{i}}{k t}-\frac{G}{k t^{2}} & \ldots & (4.3 . g)
\end{array}
$$

3) Esquema de Anälise de Variāncia

\begin{tabular}{lcccc}
\hline Causa da Variação & G.L. & S.Q. & Q.M. & $F$ \\
\hline Tratamentos & $t-1$ & SQT & QMT & $\frac{\text { QMT }}{\text { MME }}$ \\
Efeitos Residuais & $t-1$ & SQER & QMER & $\frac{\text { QMER }}{\text { QME }}$ \\
Erro & $(k t-2) t+1$ & SQE & QME & \\
\hline o $t$ a 1 & $k t^{2}-1$ & SQ Total & & \\
\hline
\end{tabular}

onde,

$$
S Q T=\frac{1}{k t} \sum_{j} T_{j}^{2}-\frac{G^{2}}{k t^{2}} \text {. }
$$




$$
\begin{aligned}
\text { SQER } & =\frac{1}{k t} \sum_{i} R_{i}^{2}-\frac{G^{2}}{k t^{2}}, \\
\text { SQE } & =S Q \text { Total }-S Q T-\text { SQER, } \\
\text { SQ Total } & =\sum_{, i, j} y_{h i j}^{2}-\frac{G^{2}}{k t^{2}}, \\
\text { QMT } & =\frac{\text { SQT }}{t-1}, \\
\text { QMER } & =\frac{\text { SQER }}{t-1} \\
\text { QME } & =\frac{\text { SQE }}{(k t-2) t+1},
\end{aligned}
$$

4) Variância da Estimativa da Diferença entre Dois Efeitos Diretos de Tratamentos

$V\left(\hat{\tau}_{j}-\hat{\tau}_{j^{\prime}}\right)=\frac{2 \sigma^{2}}{k t}$

$(4.3 . h)$ 。

5) Variância da Estimativa da Diferença entre Dois Efeitos Residuais de Tratamentos

$$
V\left(\hat{\rho}_{i}-\hat{\rho}_{i},\right)=\frac{2 \sigma^{2}}{k t}
$$

6) Variância Média da Estimativa da Diferença entre Dois Efeitos Residuais de Tratamentos 
48.

$$
\nabla\left(\hat{p}_{i}-\hat{p}_{i},\right)=\frac{2 \sigma^{2}}{k t} \quad \ldots \quad(4 \cdot 3 \cdot j)
$$

Nota-se que estes resultados são semelhantes aos do delineamento em blocos ao acaso. 
49.

\section{CONCLUSÕES}

As conclusões deste estudo são para as Sequências Equilibradas em Série, de Tipo I, com kt > 2, nas quais o bloco hi começa com o tratamento $\underline{i}$ (ou $t+2-i$ ) e termina com o tratamento $i+1$ (ou $t+1-i), \forall h(h=1, \ldots, k)$ e $\forall i(i=1, \ldots, t)$.

5.1 - Sendo $k=1$, pode-se admitir sempre que a estrutura é do tipo $S_{1}$. Para isto, cada tratamento receberá o número do blo co no qual ele é o primeiro tratamento.

5.2 - Os parāmetros podem ser estimados através das expressões da das na seção $(4.1 .3)$.

5.3 - A anälise de variância pode ser feita como indica a seção $(4.1 .4)$.

5.4 - A variância da estimativa da diferença entre os tratamen- 
$\operatorname{tos} \tau_{j}$ e $\tau_{j}$, é dada por

$$
V\left(\hat{\tau}_{j}-\hat{\tau}_{j^{\prime}}\right]=\frac{2 \sigma^{2}}{k t}
$$

5.5 - A matriz de variâncias e covariàncias do vetor aleatório $\hat{\rho}$ pode ser obtida através de

$D(\hat{\rho})=k t \sigma^{2} L^{-1}\left(\left(t^{2}-t-2,-t+1,-t, \ldots,-t,-t+1\right)\right) L^{-1}$.

onde $\left(\left(t^{2}-t-2,-t+1,-t, \ldots,-t,-t+1\right)\right)$ é uma matriz circu lante.

5.6 - A variância mëdia da estimativa da diferença entre os efei tos residuais $\hat{\rho}_{i}$ e $\hat{\rho}_{i}$, pode ser calculada pela fórmula

$$
\nabla\left(\hat{\rho}_{i}-\hat{\rho}_{i}\right)=\frac{2 t^{2}}{t-1}\left[\frac{v_{1}}{k}-\frac{1}{k t^{3}}\right] \quad \sigma^{2} .
$$

onde $\nu_{1}$ é o elemento da diagonal da matriz

$$
\left\{\left(t^{2}-2,1,0, \ldots, 0,1\right)\right\}^{-1}
$$

Essa expressão corresponde àquela apresentada por SAMPFORD (1957), para $k=1$ ou 2 .

5.7 - Admitindo-se que não há efeitos de blocos, a análise torna-se bastante simples e os resultados são análogos aos do delineamento em blocos ao acaso, aparecendo efeitos residuais em lugar de efeitos de blocos. 
51 .

\section{SUMMARY}

From Serially Balanced Sequences, direct and residual treatment effects can be studied by a single experimental unit. We consider the following model

$$
y_{h i j}=\mu+\beta_{h i}+\tau_{j}+\rho_{\ell[h, i, j]}+\varepsilon_{h i j},
$$

where $y_{h i j}$ is the observation due to treatment $\underline{j}$ in block hi: $\tau_{j}$ is the $\underline{j}$ direct treatment effect: $\rho_{\ell(h, i, j)}$ the preceding residual treatment effect over treatment $\underline{j}$ appearing in block hi, and $\varepsilon_{h i j}$ is identical and independently normal error with mean 0 and variance $\sigma^{2}$.

Following classical developments we found:

1. Normal equations.

2. Parameters estimators.

3. Decomposition of Total Sum of Squares. 
4. Variance of the difference of two means.

5. Covariance matrix of residual treatment effects.

6. Mean Variance of the estimate of the difference between two residual effects.

When block effects are absent, the analysis is rather simplified, and we can analyse as if we had randomized block design, with residual effects sum of squares in place of block sum of squares. 


\section{LITERATURA CITADA}

FINNEY, D.J。e A.D. OUTHWAITE, 1955. Serially Balanced Sequences。 Nature. Londres, 176: 748.

FINNEY, D.J。e A.D。OUTHWAITE, 1956. Serially Balanced Sequences in Bioassay. Proceedings of the Royal Society, Série B. Londres, 145: 493-507。

600D, I.J., 1950. On the Inversion of Circulant Matrices. Biometrika. Londres, 37: 185-186。

SAMPFORD, MoR., 1957. Methods of Construction and Analysis of Serially Balanced Sequences. Journal of the Royal Statistical Society. Série B. Londres, 19: 286-304.

SINHA, B.K。, 1971. Contribution to Comparison of Experiments: Optimum Experiments for Linear Inference. Calcutta, India, Universida de de Calcutta, 204 p. (Tese). 